\title{
The chemotherapeutic CX-5461 primarily targets TOP2B and exhibits selective activity in high-risk neuroblastoma
}

Min Pan ${ }^{1}$, William C. Wright ${ }^{1}$, Richard H. Chapple ${ }^{1}$, Asif Zubair ${ }^{1}$, Manbir Sandhu ${ }^{2}$, Jake E. Batchelder ${ }^{2,3}$, Brandt C. Huddle (10 4, Jonathan Low ${ }^{3}$, Kaley B. Blankenship ${ }^{5,6}$, Yingzhe Wang (1) 7 , Brittney Gordon ${ }^{6}$, Payton Archer ${ }^{5,6}$, Samuel W. Brady (1) 1, Sivaraman Natarajan (10 1, Matthew J. Posgai ${ }^{8}$, John Schuetz ${ }^{9}$, Darcie Miller ${ }^{2}$, Ravi Kalathur (10 2, Siquan Chen ${ }^{10}$, Jon Patrick Connelly (1) ${ }^{11}$, M. Madan Babu (1) 2, Michael A. Dyer (1D 6,12, Shondra M. Pruett-Miller (1) ${ }^{11}$, Burgess B. Freeman III7, Taosheng Chen (1) ${ }^{3}$, Lucy A. Godley (10) ${ }^{8}$, Scott C. Blanchard (1) ${ }^{2,3}$, Elizabeth Stewart (iD ${ }^{5,6}$, John Easton (i) ${ }^{1 凶}$ \& Paul Geeleher (i) ${ }^{1 凶}$

Survival in high-risk pediatric neuroblastoma has remained around 50\% for the last 20 years, with immunotherapies and targeted therapies having had minimal impact. Here, we identify the small molecule CX-5461 as selectively cytotoxic to high-risk neuroblastoma and synergistic with low picomolar concentrations of topoisomerase I inhibitors in improving survival in vivo in orthotopic patient-derived xenograft neuroblastoma mouse models. CX-5461 recently progressed through phase I clinical trial as a first-in-human inhibitor of RNA-POL I. However, we also use a comprehensive panel of in vitro and in vivo assays to demonstrate that CX-5461 has been mischaracterized and that its primary target at pharmacologically relevant concentrations, is in fact topoisomerase II beta (TOP2B), not RNA-POL I. This is important because existing clinically approved chemotherapeutics have well-documented offtarget interactions with TOP2B, which have previously been shown to cause both therapyinduced leukemia and cardiotoxicity-often-fatal adverse events, which can emerge several years after treatment. Thus, while we show that combination therapies involving CX-5461 have promising anti-tumor activity in vivo in neuroblastoma, our identification of TOP2B as the primary target of $\mathrm{CX}-5461$ indicates unexpected safety concerns that should be examined in ongoing phase II clinical trials in adult patients before pursuing clinical studies in children.

\footnotetext{
${ }^{1}$ Department of Computational Biology, St. Jude Children's Research Hospital, Memphis, TN 38105, USA. ${ }^{2}$ Department of Structural Biology, St. Jude Children's Research Hospital, Memphis, TN 38105, USA. ${ }^{3}$ Department of Chemical Biology and Therapeutics, St. Jude Children's Research Hospital, Memphis, TN 38105, USA. ${ }^{4}$ The Department of Physiology and Biophysics, Weill Cornell Medicine, New York, NY 10065, USA. ${ }^{5}$ Department of Oncology, St. Jude Children's Research Hospital, Memphis, TN 38105, USA. ${ }^{6}$ Department of Developmental Neurobiology, St. Jude Children's Research Hospital, Memphis, TN 38105, USA. ${ }^{7}$ Preclinical Pharmacokinetic Shared Resource, St. Jude Children's Research Hospital, Memphis, TN 38105, USA. ${ }^{8}$ Departments of Medicine and Human Genetics, The University of Chicago, Chicago, IL 60637, USA. ${ }^{9}$ Department of Pharmaceutical Sciences, St. Jude Children's Research Hospital, Memphis, TN 38105, USA. ${ }^{10}$ Cellular Screening Center, The University of Chicago, Chicago, IL 60637, USA. ${ }^{11}$ Department of Cell and Molecular Biology, St. Jude Children's Research Hospital, Memphis, TN 38105, USA. ${ }^{12}$ Howard Hughes Medical Institute, Chevy Chase, MD 20815, USA. 凶email: john.easton@stjude.org; paul.geeleher@stjude.org
} 
$\mathrm{N}$ euroblastoma is a pediatric cancer of the developing peripheral nervous system and the most common solid tumor in children ${ }^{1}$. Pediatric cancers have different mutation profiles compared to adult cancers, typically exhibiting far fewer targetable oncogene mutations ${ }^{2}$. Additionally, the low mutation burden and resulting lack of neoantigens means immunotherapies have had only a modest impact ${ }^{3}$. Consequently, the overall survival in high-risk neuroblastoma has remained around $50 \%$ for the past 20 years ${ }^{4}$, meaning a promising small molecule inhibitor is of tremendous interest to treat this devastating disease.

Since the release of the Cancer Cell Line Encyclopedia (CCLE) ${ }^{5}$ and the Genomics of Drug Sensitivity in Cancer (GDSC) ${ }^{6}$, large drug screening datasets in cancer cell lines have emerged as a valuable resource for identifying new therapeutic strategies to treat pediatric cancers, facilitating discoveries that could not have been made based on mutation profiles alone. For example, these datasets provided the original evidence that PARP inhibitors would be effective treating Ewing's Sarcoma, which has since proven to have clinical activity in combination with DNA damaging agents ${ }^{7,8}$. Perturbational screens in these cell lines have also motivated the development of EZH2 inhibitors in pediatric rhabdoid tumors ${ }^{9}$ and BRD4 inhibitors in neuroblastoma ${ }^{10,11}$. However, the rapid growth of these datasets ${ }^{12-14}$ means systematic interrogation of the pediatric data has not been carried out, limiting the potential for prioritizing promising targets in these diseases.

Here, we mined these datasets to identify an unexpected, reproducible, selective effect of the compound CX-5461 in highrisk pediatric neuroblastoma. Motivated by the striking drug screening results, we performed a comprehensive study in orthotopic patient-derived xenograft (PDX) bearing mice to show that CX-5461 has anti-neuroblastoma activity in vivo at pharmacologically relevant dosages derived using an in vivo pharmacokinetics modeling approach. CX-5461 recently progressed through phase I clinical trial and is most widely accepted as a first-in-human selective inhibitor of RNA-POL I ${ }^{15}$. Here, we also comprehensively show that CX-5461 is mischaracterized and its primary target at physiological concentrations is in fact TOP2B, which because of this gene's unique functional role, has potentially important clinical implications for patient stratification, drug combinations, and likely side-effect profiles. Critically, $T O P 2 B$ is highly expressed in some normal cells and off-target drug interactions with this gene have previously been implicated in late-emerging therapy-induced acute leukemias ${ }^{16,17}$ and cardiotoxicity $^{18}$, which often lead to death. Thus, while CX-5461 has promising anti-tumor activity in vivo, our results indicate that it also has the potential to cause previously unanticipated patient harm, which should be investigated in adults before clinical studies are proposed in children.

\section{Results}

CX-5461 exhibits selective and potent activity against neuroblastoma cell lines. We first devised a quantitative metric to identify compounds with selective activity against neuroblastoma cell lines in large drug screening datasets (similar to Durbin et al. ${ }^{10}$ ). Specifically, for each drug, we ranked cell lines by their $\mathrm{IC}_{50}$, identified the rank of the median neuroblastoma cell line, and then normalized this value to a $0-1$ scale by dividing by the total number of cell lines screened against this drug-we refer to this as a "Selectivity Score". We calculated Selectivity Scores for each drug in the most recent release of the Sanger Institute's GDSC cell line drug screening dataset, where a total of 265 drugs were screened against 1,001 cancer cell lines ${ }^{19}$, including 31 neuroblastoma cell lines, the most of currently available datasets. Drugs with the highest
Selectivity Score included several already in investigation in neuroblastoma (Fig. 1A; Supplementary Data Table S1), for example, ranked \#3 and \#4 were IGF1R inhibitors (linsitinib and BMS$754807)^{20}$. Surprisingly, the second most selective drug for neuroblastoma was CX-5461, a compound that recently completed phase I clinical trial as a first-in-human selective inhibitor of RNA-POL I, but is not currently in clinical investigation in neuroblastoma ${ }^{4}$. The selective activity was striking (Fig. 1B, Fig. S1A, $P=5.2 \times 10^{-6}$ from t-test of neuroblastoma $\mathrm{IC}_{50}$ values vs all other cell lines) with almost half of neuroblastoma cell lines achieving an $\mathrm{IC}_{50}$ in the nanomolar range. We obtained and rescreened 8 of the GDSC cell lines with CX-5461 and achieved highly consistent results (Fig. 1C, $r=0.94$, $P=5.7 \times 10^{-4}$; Fig. S1B, C). Additionally, CX-5461 was included in PRISM, a recent independent study ${ }^{13}$ that screened 4,518 drugs against 578 cancer cell lines using a new high-throughput multiplex sequencing-based assay. Although only 10 neuroblastoma cell lines were included, neuroblastoma remained the second most sensitive group to CX-5461 among the 24 cancer types screened (Fig. 1D). The overall trends for Selectivity Scores across drugs were also recapitulated in PRISM (Fig. 1E; Fig. S1D), with CX-5461 remaining among the most selective drugs for neuroblastoma cell lines.

To deduce whether CX-5461 may favor specific subclasses of neuroblastoma, we next sought to identify genetic predictors of CX-5461 response in these neuroblastoma cell lines. Using the GDSC's exome sequencing data and consistent with previous reports $^{21,22}$ we identified MYCN amplification and TP53 mutation as independently predictive of response $\left(P=6.4 \times 10^{-4}\right.$ from a multivariate linear model; $R^{2}=0.4$, Fig. $\left.1 \mathrm{~F}\right)$. The effect sizes were large; TP53 mutation was associated with a 17.45 \pm 4.7 (mean effect $+/-95 \% \mathrm{CI}$ ) fold increase in $\mathrm{IC}_{50}$, while $M Y C N$ amplification was associated with a $10.9 \pm 5.6$-fold decrease in $\mathrm{IC}_{50}$. We tested these predictions in vitro using short hairpin RNA (shRNA) knockdown of $M Y C N(P<0.05$ for each of three independent shRNAs, Fig. 1G, H) and CRISPR knockout of TP53 $\left(P=2.3 \times 10^{-3}\right.$, Fig. 1I) in CHP-134 (MYCN amp, TP53 wt.) neuroblastoma cells, identifying a causal relationship with CX$5461 \mathrm{IC}_{50}$ in both cases. We recalculated the Selectivity Scores for only MYCN amplified TP53 wild-type neuroblastoma cell linesrepresentative of high-risk disease-where CX-5461 was the \#1 most selective compound for these cell lines (Fig. S1E, S1F). Thus, CX-5461 is of particular interest in the high-risk subgroup of neuroblastoma, where $M Y C N$ amplification is the primary driver and patient outcomes are extremely poor.

CX-5461 kills neuroblastoma cells at low concentrations at which inhibition of RNA-POL I was not detected. CX-5461 recently completed phase I clinical trial as a selective inhibitor of RNA-POL I ${ }^{15}$. To confirm that this was indeed the mechanismof-action against neuroblastoma cells, we performed a standard assay to determine whether CX-5461 interferes with RNA-POL I activity $^{21}$ - using $\mathrm{qPCR}$ to the short-lived internal transcribed spacer (ITS) region of the $45 \mathrm{~S}$ rRNA transcript. We applied this in CX-5461-treated CHP-134 and IMR-5 (both highly CX-5461 sensitive) neuroblastoma cell lines. Surprisingly, we observed no detectable inhibition of RNA-POL I activity in these cell lines at the low sub-micromolar concentrations at which CX-5461 was effective (Fig. 1J and S1G, H). However, in four CX-5461resistant neuroblastoma cell lines, where higher CX-5461 concentrations could be tolerated, we observed inhibition of RNAPOL I (Fig. 1J). This is consistent with RNA-POL I inhibition emerging either as an off-target effect or as a consequence of inhibiting some unknown drug target. We validated these observations using a 5-ethynyl uridine (EU) incorporation assay, where again there was no detectable inhibition of RNA synthesis at the low concentrations of CX-5461 that were effective against 


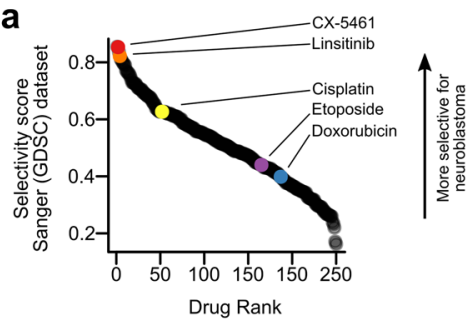

b
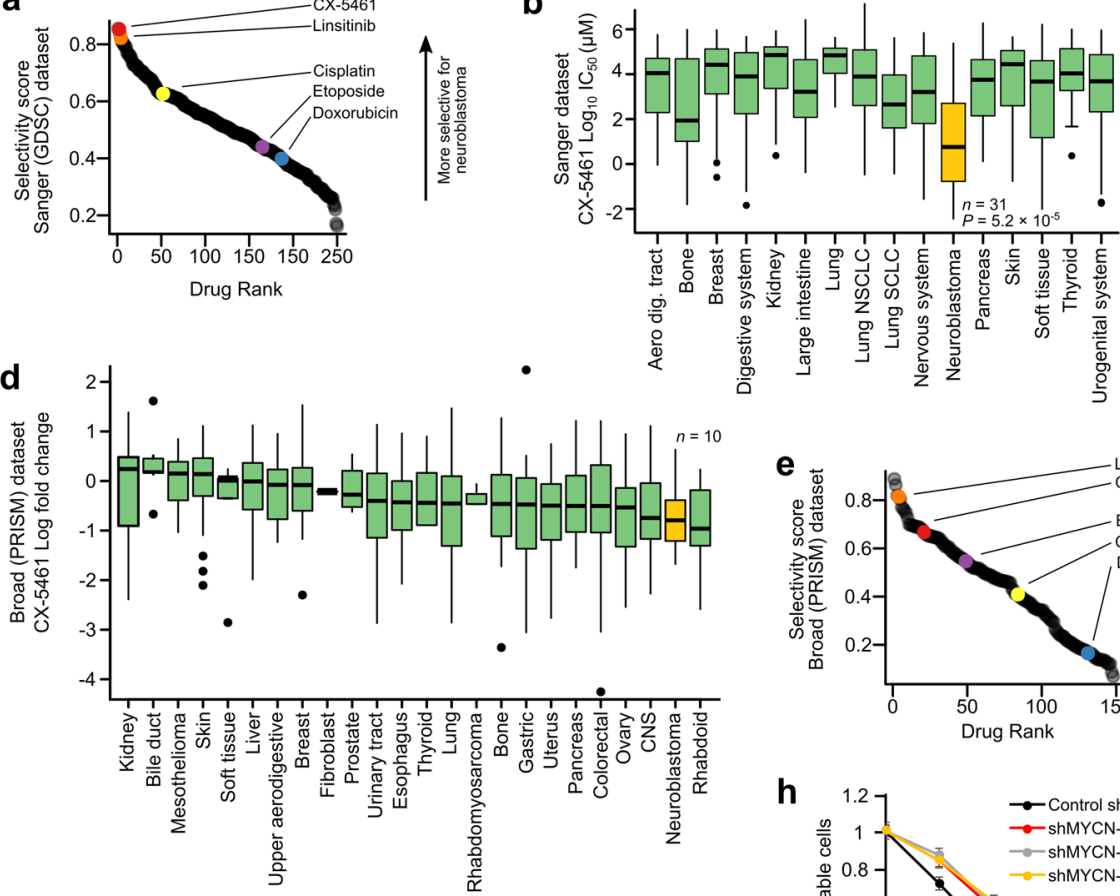

g

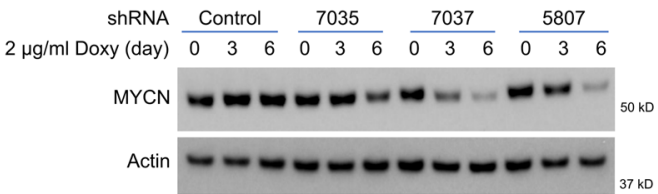

j

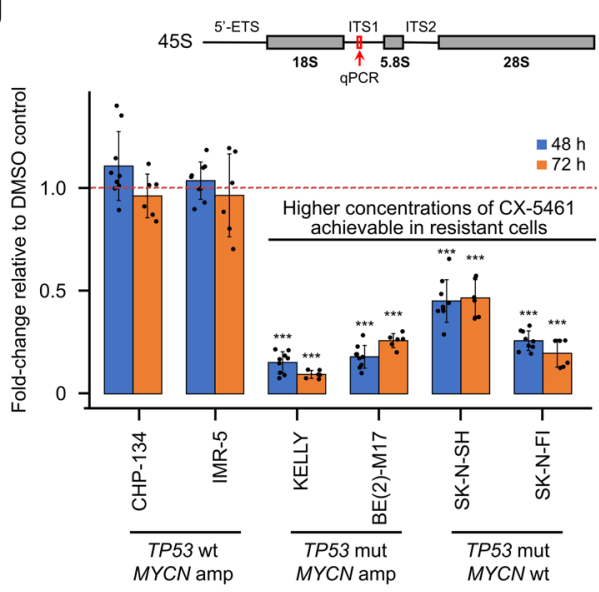

k

k
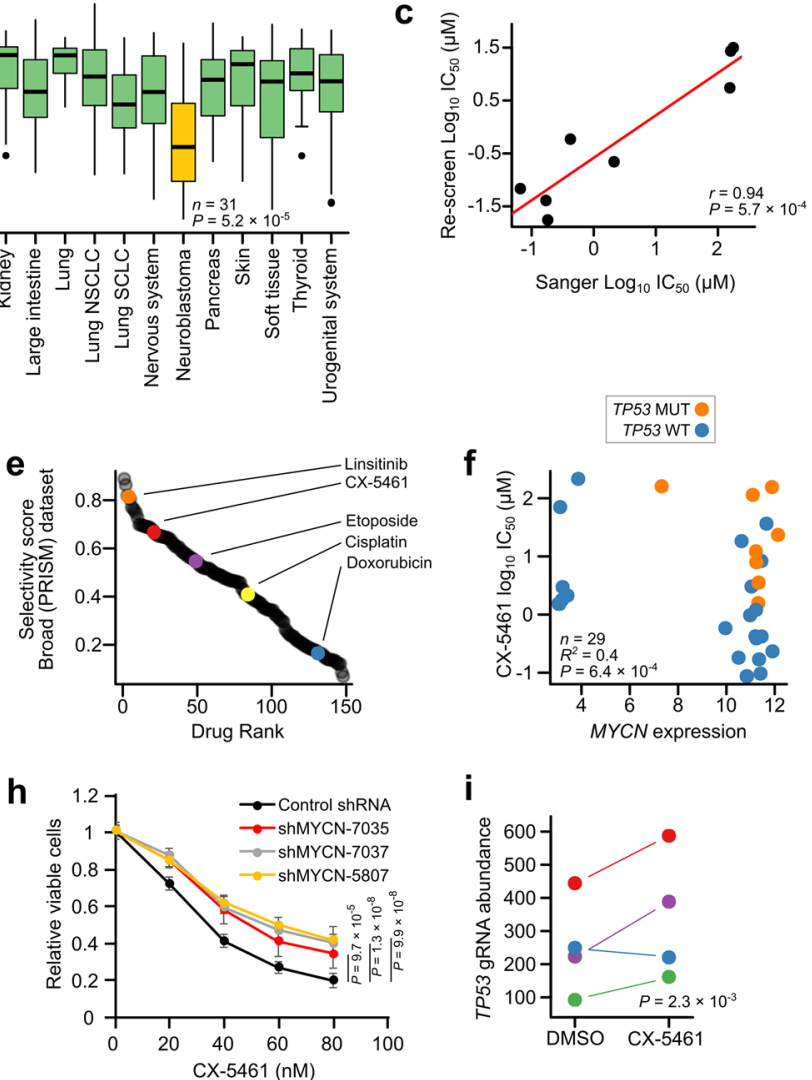

i


m

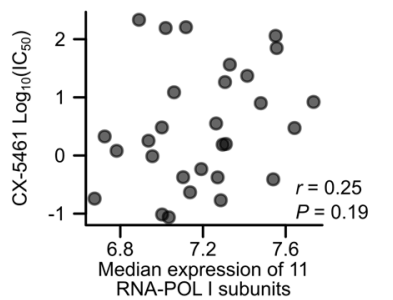

CHP-134 and IMR-5 cells (Fig. 1K). The resistant cell line KELLY, which could tolerate higher concentrations of CX-5461, again showed clear inhibition of RNA-POL I. RNA POL I inhibition induced by CX-5461 was observed as quickly as $2 \mathrm{~h}$ after treatment in KELLY cells, whereas CHP-134 showed no change of RNA-POL I activity in the time period from 2 to $24 \mathrm{~h}$ (Fig. S1I). We also identified no significant associations between the baseline expression levels of RNA-POL I complex genes and CX-5461 $\mathrm{IC}_{50}$ in GDSC neuroblastoma cell lines (Fig. 1L) or between the median expression of RNA-POL I complex genes and response to CX-5461 (Fig. 1M). Finally, treatment with CX-5461 for 48 or $72 \mathrm{~h}$ had no apparent impact on levels of mature cellular rRNA in neuroblastoma cells (Fig. S1J, K). These results indicate that CX-5461's anti-cancer activity may not exclusively result from direct selective inhibition of
RNA-POL I. Instead, these findings suggest that CX-5461 may be active against neuroblastoma cell lines by one or more additional mechanisms.

CX-5461 is a topoisomerase II inhibitor. The genome-wide CRISPR knockout screen has emerged as a powerful, unbiased assay for determining the mechanism-of-action of small molecules ${ }^{23}$. The workflow involves culturing cells stably expressing Cas9 with or without the drug of interest, performing genome-wide CRISPR gene knockout, and comparing the relative abundance of different guide RNAs in drug-treated vs control cells; this acts as a proxy for the proportion of surviving cells that have undergone each gene knockout (Fig. 2A). We performed such a screen in CHP-134 neuroblastoma cells treated with $0.04 \mu \mathrm{M}$ CX-5461 or DMSO control, allowing us to identify genes 
Fig. 1 Neuroblastoma cell lines are selectively sensitive to CX-5461. a Summary score representing selectivity for neuroblastoma cell lines plotted for each of 265 drugs screened by GDSC. $\mathbf{b} \log _{10}\left(I_{50}\right)$ values for CX-5461 for all non-hematological cancer cell lines screened by GDSC (see Fig. S1A). P-value is from a two-sided t-test. $\mathbf{c} \log _{10}\left(\mathrm{IC}_{50}\right.$ ) values for CX-5461 for eight cell lines screened by GDSC and re-screened in this study. $\mathbf{d}$ Log ( $_{10}$ (fold change) values for CX5461 for all cell lines screened by PRISM. A lower fold-change implies higher drug effectiveness. e Summary score representing selectivity for neuroblastoma cell lines plotted for each of 148 drugs screened in PRISM (shown for drugs screened by both PRISM and GDSC). $\mathbf{f}$ MYCN expression level and TP53 status against $\log _{10}\left(\mathrm{IC}_{50}\right)$ values for $\mathrm{CX}-5461$ in GDSC. P-value from multivariate linear regression. $\mathbf{g}$ MYCN protein levels following MYCN knockdown in CHP-134 cells. $\beta$-Actin was used as loading control. Representative results of three biological replicates are shown. Doxy, doxycycline. $\mathbf{h}$ Viability of CHP-134 cells following MYCN knockdown measured with MTS. Data represent mean \pm SD of $n=10$ biological replicates. i Relative abundance of four independent TP53 guide RNAs in a genome-wide CRISPR screen in either a DMSO or CX-5461 treated CHP-134 cells. j Pre-rRNA 45 S expression in CX-5461 treated cell lines relative to DMSO, determined by RT-qPCR with primers located in an internal transcribed spacer (ITS) region of the rRNA transcript. Data represent mean \pm SD of $n=9(48 \mathrm{~h})$ or $n=6(72 \mathrm{~h})$ biological replicates. CX-5461 concentration and $P$ value (left to right): CHP-134, 0.2 $\mu$ M, 0.091, 0.49; IMR-5, $0.05 \mu \mathrm{M}, 0.49,0.67 ; \mathrm{KELLY}, 2 \mu \mathrm{M}, 8.9 \times 10^{-13}, 5.9 \times 10^{-11} ; \mathrm{BE}(2)-\mathrm{M} 17,10 \mu \mathrm{M}, 2.9 \times 10^{-13}, 1.8 \times 10^{-9} ; \mathrm{SK}-\mathrm{NSH}, 2 \mu \mathrm{M}, 8.2 \times 10^{-9}, 4.9 \times 10^{-6} ; \mathrm{SK}-\mathrm{N}-\mathrm{Fl}$, $20 \mu \mathrm{M}, 1.1 \times 10^{-11}, 9.8 \times 10^{-9} . \mathbf{k}$ EU incorporation assay to assess global nascent RNA transcription. Representative results of three biological replicates are shown. CX-5461 concentration: CHP-134, $0.2 \mu \mathrm{M}$; IMR-5 $0.05 \mu \mathrm{M} ; \mathrm{KELLY}, 2 \mu \mathrm{M}$. Scale bar $=20 \mu \mathrm{m}$. I Spearman's correlation of the expression of all genes in GDSC with the reciprocal of CX-5461 IC 50 ( $y$-axis) in 29 neuroblastoma cell lines. Genes unique to the RNA-POL I complex are highlighted in red. $P$-value is from two-sided paired t-test. $\mathbf{m}$ Correlation of the median expression level of 11 genes of the RNA-POL I complex ( $x$-axis) with CX-5461 log ${ }_{10}\left(I_{50}\right)(y$-axis) in 29 GDSC neuroblastoma cell lines. P-value is from Two-sided Pearson's correlation test. In all boxplots, the center line represents the median, the bound of box is upper and lower quartiles and whiskers are $1.5 \times$ the interquartile range. Two-tailed t-test was used for (h and $\mathbf{j})$. Source data for (h and $\mathbf{j})$ are included in Source data file.

required for the growth of CHP-134 cells in the presence of CX5461. Notably, none of the top hits had any direct relationship to RNA-POL I, however, there was a clear enrichment of genes related to DNA-damage response (Fig. $2 \mathrm{~B}$ and Fig. S2A, B). Remarkably, all three of the most significant knockouts had a direct relationship to topoisomerase II (Fig. 2C-E): TDP2 catalyzes the removal of the covalent bonds between topoisomerase II and $\mathrm{DNA}^{24}$, LIG4 is a DNA ligase that rejoins double-strand breaks caused during normal topoisomerase II activity ${ }^{25}$, and inhibition of FZR1 has previously been shown to sensitize cells to topoisomerase II inhibitors ${ }^{26}$. We queried a recently published resource ${ }^{23}$ that performed CRISPR knockout screens in human retinal epithelial cells treated with 31 DNA damaging agents and confirmed that TDP2, LIG4, and FZR1 knockouts all sensitize these cells to the known topoisomerase II inhibitors doxorubicin and etoposide (Fig. 2F, G). These results suggest that CX-5461's primary target at pharmacologically relevant concentrations is in fact topoisomerase II. Such a mode of action seems especially plausible given that CX-5461 is a fluoroquinolone derivative ${ }^{27}$ and maintains structural similarity to these antibiotics, which target bacterial DNA gyrase-an ortholog of human topoisomerase $\mathrm{II}^{28}$.

To investigate further whether CX-5461's structure is consistent with that of a topoisomerase II inhibitor, we performed molecular dynamics simulations using existing TOP2A and TOP2B protein structures. The in silico binding of CX-5461 parelleled the binding mechanism of classical topoisomerase II poisons, including fluoroquinolones, etoposide, and doxorubicin, where the drug traps the topoisomerase protein covalently bound to DNA, leading to DNA damage and cell death ${ }^{29}$. CX-5461 interacts with the DNA breakpoint, collectively surrounded by the TOP2A/B DNA binding domains (Fig. 2H; Fig. S2C, D). We ran each simulation for 10 nanoseconds (Fig. S2E) and found that CX-5461 interactions differ between TOP2A and TOP2B, interacting with largely non-analogous amino acids of the respective proteins (Fig. 2I; Fig. S2F). We calculated the relative binding affinity of the CX-5461 ligand to the TOP2A/B proteins and noted a slightly increased affinity of the ligand to TOP2B. (Fig. 2J).

We next performed several orthogonal experimental assays to test the hypothesis that CX-5461 is a topoisomerase II poison. We first performed a band depletion assay ${ }^{30}$, to assess the stabilization of TOP2-DNA covalent complexes (called the TOP2 cleavable complex (TOP2cc)), following CX-5461 treatment. This assay showed a clear depletion of free TOP2A and TOP2B protein, consistent with these proteins being trapped to DNA following treatment with CX-5461 (Fig. 2K, Fig. S2G, H). The decrease of free TOP2 was dose-dependent and comparable to the positive control TOP2 poison doxorubicin (Fig. 2L, Fig. S2G, H). The cisplatin-treated negative control showed no change (Fig. 2M). CX-5461 did not affect TOP1 (Fig. 2K), suggesting its activity is selective to TOP2. We next used a complementary assay, the rapid approach to DNA adduct recovery (RADAR) assay, to further assess the ability of CX-5461 to form TOP2 $\mathrm{cc}^{31}$. This revealed CX-5461 treatment-induced TOP2cc formation, for both TOP2A and TOP2B, in all four neuroblastoma cell lines tested (Fig. S2I), again consistent with CX-5461 trapping TOP2 to DNA and behaving as a classical topoisomerase II poison.

Sub-micromolar concentrations of CX-5461 induce cell cycle arrest and kill neuroblastoma cells by causing DNA damage and cell death by apoptosis. The mechanism of drug-induced cell death by classical topoisomerase II poisons, such as etoposide and doxorubicin is well understood. These drugs trap topoisomerase protein to DNA, and these DNA-topoisomerase complexes are converted to DNA double-strand breaks when encountered by the DNA replication or transcription machinery, leading to cell death by apoptosis ${ }^{32}$.

To investigate whether this was the mechanism of cell death in neuroblastoma cells susceptible to sub-micromolar concentrations of CX-5461, we first used flow cytometry to quantify cells staining positive for annexin $\mathrm{V}$ and propidium iodide (PI), canonical markers of apoptosis and cell death respectively, following CX-5461 treatment at $0.1,0.2$ and $0.5 \mu \mathrm{M}$ over $48 \mathrm{~h}$. These assays showed that CX-5461 was causing cell death by apoptosis at these low concentrations in CX-5461 sensitive CHP134 and IMR-5 neuroblastoma cells (Fig. 3A-C). Induction of cell death by apoptosis at low concentrations of CX-5461 was confirmed by Western blot for cleaved PARP (c-PARP, Fig. 3D) in CHP-134 and IMR-5 cell lines. In the resistant cell lines KELLY and BE(2)-M17, $0.5 \mu \mathrm{M}$ of CX-5461 was sufficient to induce apoptosis (Fig. S3A).

We next investigated CX-5461's DNA damaging effects, using immunofluorescence for canonical DNA damage markers $\gamma$ H2AX, 53BP1 (Fig. 3E, Fig. S3B-D), and RPA (Fig. S3E) in CHP134 and IMR-5 cell lines. Indeed, these markers were evident as early as $2 \mathrm{~h}$ following treatment with nanomolar concentrations of CX-5461. We confirmed DNA damage using a single-cell alkali 


\section{a}
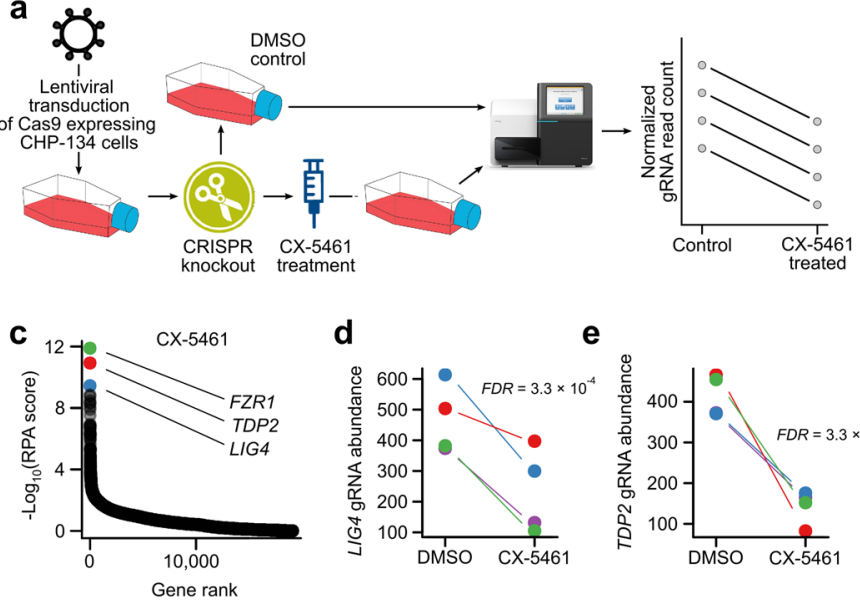

e



h
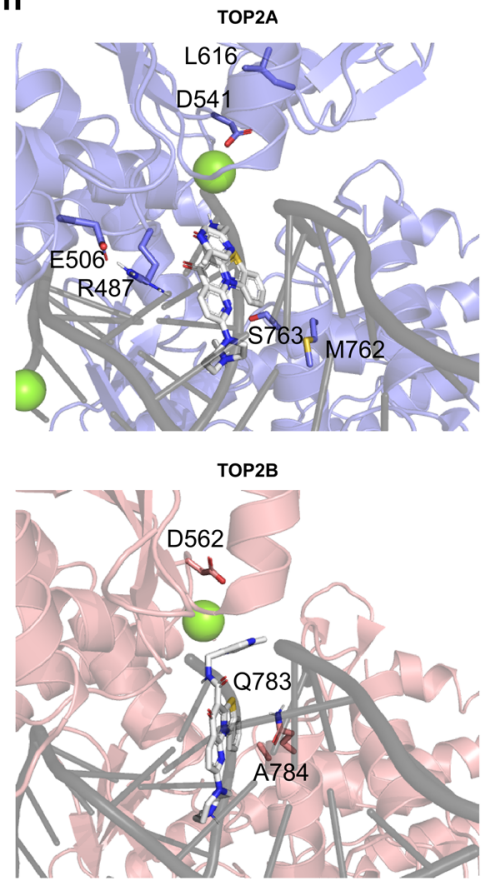
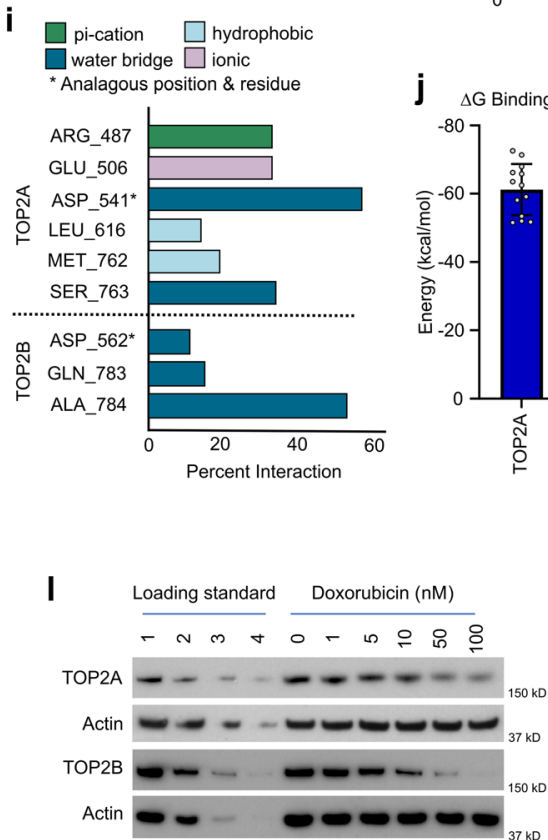

b
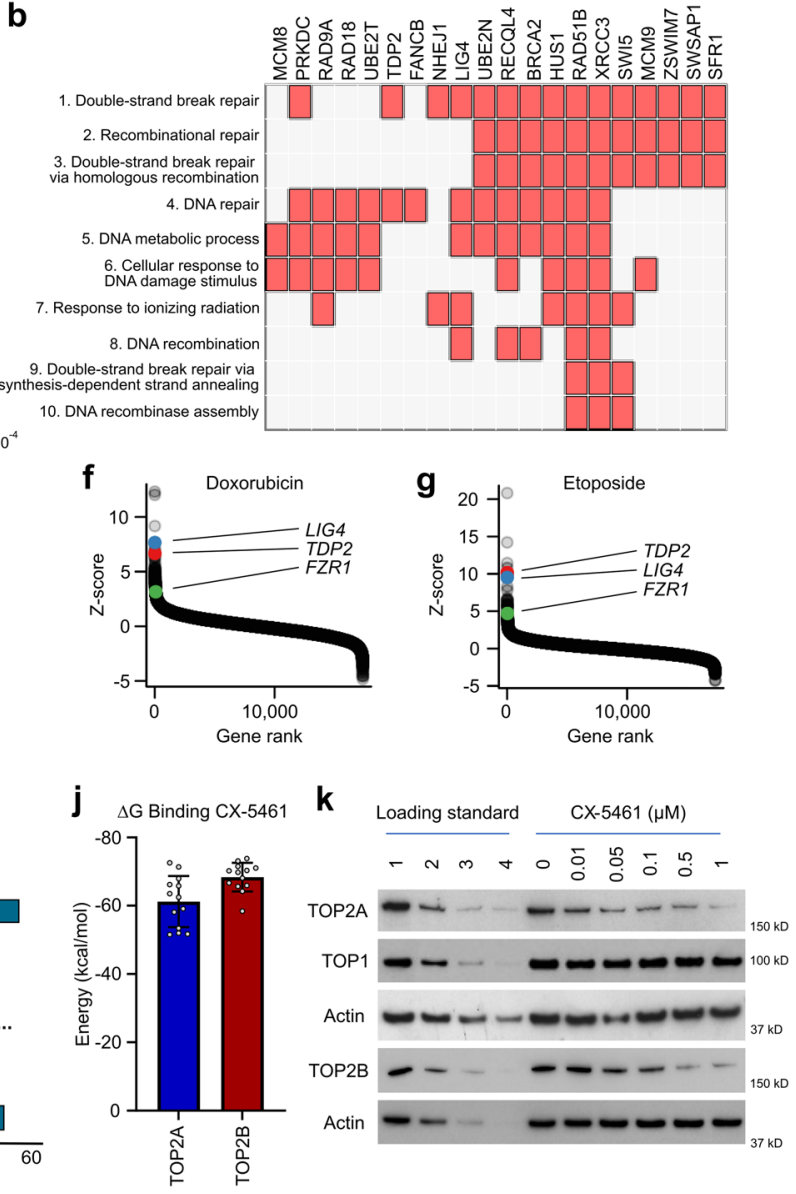

Fig. 2 CX-5461 is a topoisomerase II inhibitor. a Schematic of CRISPR knockout screening strategy. b Clustergram generated by Enrichr showing the assignment of statistically significant (red boxes) gene knockouts to the most highly enriched GO (2018) Biological Processes. Genes with an FDR < 0.05 $(n=40)$ were used as input. c Top hits in our genome-wide CRISPR knockout screen in CHP-134 cells. Genes are ranked by -log 10 (RPA score). d Relative abundance of 4 independent LIG4 guide RNAs in our CRISPR screen. e Like (d) but for TDP2. f Waterfall plot summarizing CRISPR knockout screening data obtained from Oliveri et al. Higher values on the $y$-axes imply that knockout has sensitized cells to doxorubicin. TDP2, LIG4, and FZR1 are highlighted. $\mathbf{g}$ Like (f) but results shown for the topoisomerase II inhibitor etoposide. $\mathbf{h}$ Representative poses from equilibrated molecular dynamics simulations of CX-5461 bound to TOP2A (top) or TOP2B (bottom). DNA strands are shown in gray. CX-5461 is displayed in white, and its heteroatoms colored blue (nitrogen), red (oxygen), and sulfur (yellow). Green represents magnesium ions. i Percentage of CX-5461 interactions with TOP2A or TOP2B in simulations. Residues interacting with either protein are listed if they conferred $\geq 10 \%$ interaction with $C X-5461$. Bar colors correspond to the type of interaction. ( ${ }^{\star}$ ) indicates residues in the same position when the two proteins are superimposed. $\mathbf{j}$ Weighted average of the relative binding affinity for $\mathrm{CX}-5461$ is plotted for TOP2A and TOP2B. Data represent mean \pm SD of $n=13$ independent weighted simulation clusters (described in "Methods"). $P=0.01$ from two-sided t-test. The source data are included in Source data file. Band-depletion assay for CX-5461 (k), doxorubicin (I), and cisplatin (m) treated CHP-134 cells. For lanes 1-4, protein equal to $33,16.5,8.3$, and 4.1 thousand untreated cells were loaded respectively. For each of the rest of the lanes, protein equal to 33 thousand cells was loaded. Representative results of three biological replicates are shown.

gel electrophoresis (comet) assay in CHP-134 cells treated with $0.2 \mu \mathrm{M}$ CX-5461 for $30 \mathrm{~min}$ or $24 \mathrm{~h}$, with DNA damage evident at $30 \mathrm{~min}$ and increasing at $24 \mathrm{~h}$ (Fig. 3F, and Fig. S3F). Furthermore, using cell cycle analysis and an EdU incorporation assay, we found evidence of $S$ phase cell cycle arrest (Fig. 3G) and deceleration of active DNA synthesis (Fig. $3 \mathrm{H}$ ) starting $2 \mathrm{~h}$ post CX-5461 treatment. Moreover, $\gamma$-H2AX staining was heavily enriched in cells with active DNA synthesis (Fig. 3I). Together, these data suggest that DNA replication stress was induced and DNA damage was generated during DNA replication. Western blot revealed phosphorylation of cell cycle checkpoints CHK1, CHK2, and p53 (S15) in CX-5461 treated CHP-134 and IMR-5 cells, indicating activation of the DNA-damage response (Fig. S3G, H). We also found that cells surviving $24 \mathrm{~h}$ of treatment 
a


Annexin $\mathrm{V}$
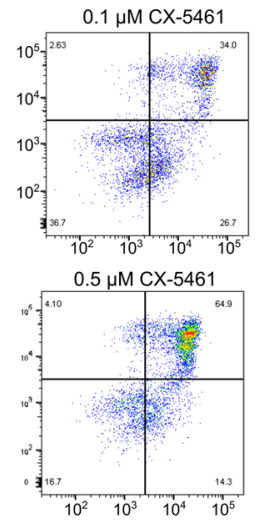

b $\quad \mathrm{CHP}-134,48 \mathrm{~h}$

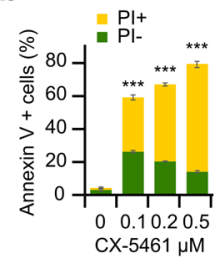

d

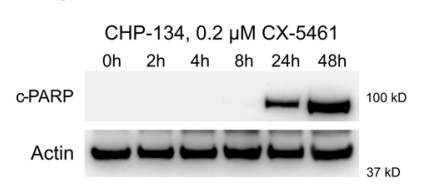

f

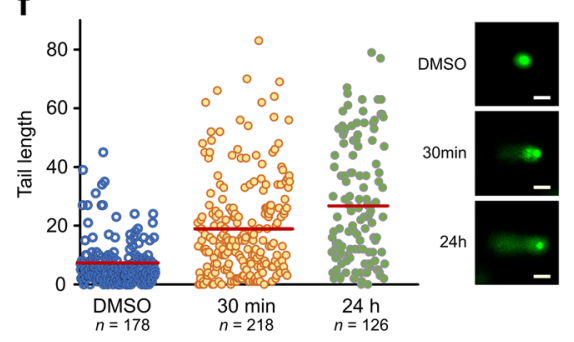

C

h g







e

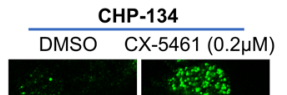

$$
\mathrm{V}-\mathrm{H} 2 \mathrm{AX}
$$

$53 \mathrm{BP} 1$
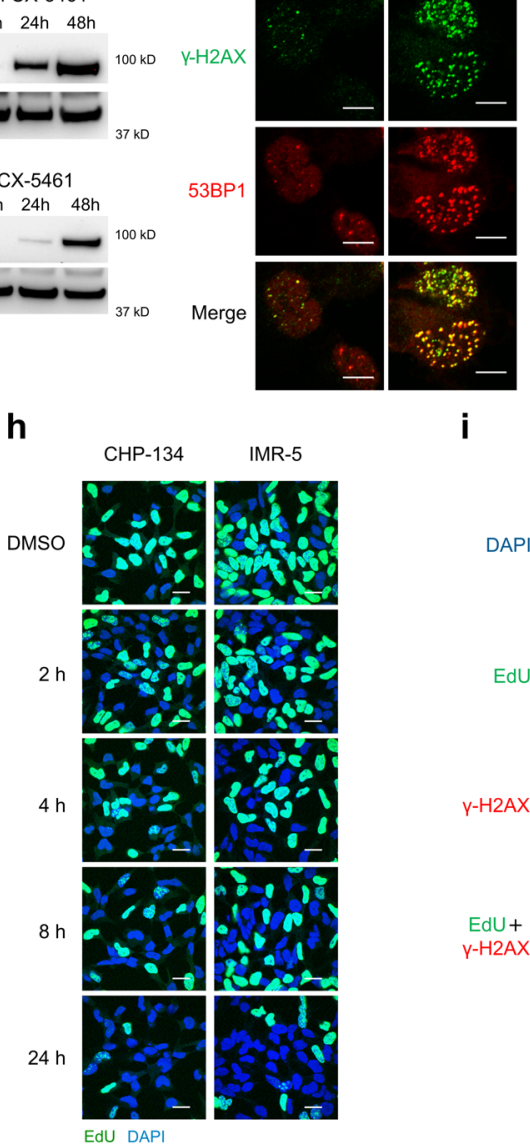
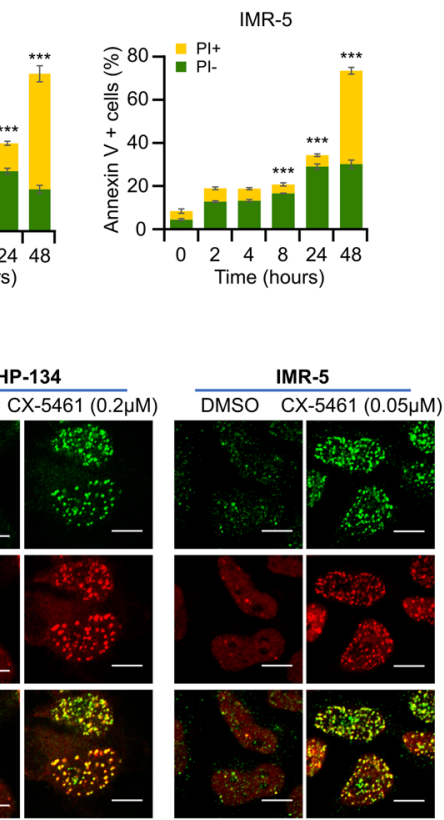

i

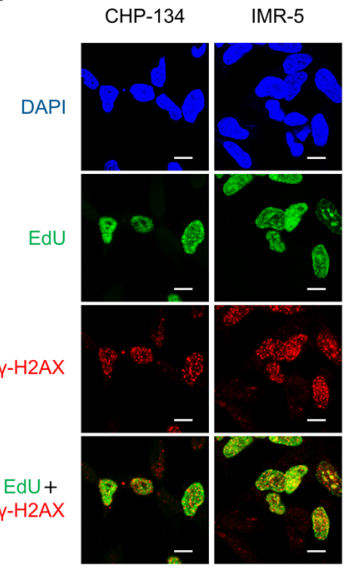

Fig. 3 CX-5461 causes DNA damage and cell death by apoptosis in neuroblastoma cells at sub-micromolar concentrations. a Apoptosis in CHP-134 cells. The cells were treated for $48 \mathrm{~h}$. $\mathbf{b}$ Quantification of data in (a). Data represent the mean $\pm \mathrm{SD}$ of three independent experiments. Significant $P$-values (left to right): $3.9 \times 10^{-6}, 7.2 \times 10^{-7}$, and $4.8 \times 10^{-7}$. c Quantification of flow cytometry data showing apoptosis up to $48 \mathrm{~h}$ in $\mathrm{CHP}-134$ and IMR-5 (0.2 $\mu \mathrm{M}$ and $0.1 \mu \mathrm{M}$ of CX-5461 respectively). CHP-134 cells were treated with $0.2 \mu \mathrm{M}$ and IMR- 5 cells with $0.1 \mu \mathrm{M}$ of CX-5461. Data represent the mean \pm SD of three independent experiments. Significant $P$-values (left to right): CHP-134, $2.2 \times 10^{-4}, 3.2 \times 10^{-5}$, and 1.5 × 10-5; IMR-5, 1.8 × 10-4, $2.3 \times 10^{-5}$, and 2.5 $\times$ $10^{-6}$. d Western blots for cleaved PARP (c-PARP). $\beta$-Actin was used as a loading control. Representative results of three biological replicates are shown. e Co-localization of 53BP1 and $\gamma-\mathrm{H} 2 \mathrm{AX}$. Cells were treated for $24 \mathrm{~h}$. Representative results of three biological replicates are shown. Scale bar $=10 \mu \mathrm{m}$. See Fig. S3d for more time points. $\mathbf{f}$ Comet assay in CHP-134 cells. Tail length (left) and representative images (right) are shown. The red line indicates the mean value of tail length in each group. Scale bar $=5 \mu \mathrm{m}$. $\mathbf{g}$ Cell cycle analysis forCHP-134 (0.2 $\mu$ M CX-5461) and IMR-5 (0.05 $\mu$ M CX-5461) cells. Data represent the mean $\pm S D$ of three independent experiments. $P$-values vs $0 \mathrm{~h}: P 1=4.2 \times 10^{-5}, P 2=7.1 \times 10^{-7}, P 3=6.3 \times 10^{-5}, P 4=2.7 \times 10^{-4}, P 5=6.3 \times$ $10^{-3}, P 6=2.3 \times 10^{-5}, P 7=1.8 \times 10^{-4}$, and $P 8=0.044$. h EdU incorporation assay to assess global nascent DNA transcription. Representative results of three biological replicates are shown. Blue, DAPI; green, EdU. Scale bar $=20 \mu \mathrm{m}$. i CX-5461 induced DNA damage $(\gamma-\mathrm{H} 2 \mathrm{AX}$, red) was enriched in cells with active DNA synthesis (positive for EdU (green) labeling). Representative results of three biological replicates are shown. Scale bar $=10 \mu \mathrm{m}$. Two-tailed ttest was used for $(\mathbf{b}, \mathbf{c}, \mathbf{g})$. Source data for these panels are included in Source Data file.

with CX-5461 accumulated in the G2/M phase of cell cycle (Fig. 3G), consistent with arrested cells undergoing DNA damage repair. Furthermore, CX-5461 induced DNA damage was diminished by MYCN knockdown and TOP2A and TOP2B expression were also suppressed (Fig. S3I, J). Hence, the DNA damaging effects of low sub-nanomolar concentrations of CX-
5461 in proliferating cells follow the hallmark DNA-damaging activities of topoisomerase II poisons.

CX-5461 primarily targets the TOP2B paralog. The findings above initially seemed to be consistent with a single isolated 
study, which used a computational systems biology approach to predict CX-5461 as a TOP2A poison ${ }^{33}$. This is exciting as TOP2A is one of the most successful drug targets in cancer ${ }^{34}$; however, the consideration of TOP2A as CX-5461's primary target has critical inconsistencies. Why did other topoisomerase II poisons (etoposide, doxorubicin) not also show selective activity against neuroblastoma cell lines (Fig. 1A, E)? Moreover, why has CX5461 been shown to inhibit RNA-POL I in many different cell types, including non-cycling cells ${ }^{35}$, which do not express TOP $2 \mathrm{~A}^{36}$ ? Consistent with our molecular dynamic simulations, the answers appear to stem from CX-5461's preferential interaction with TOP2B - which despite structural similarity is functionally distinct from TOP $2 \mathrm{~A}^{16}$, a distinction that has potential clinical implications for this compound.

We first observed that although TOP2A and TOP2B were both overexpressed in neuroblastoma cell lines (Fig. S4A, B), the magnitude of $T O P 2 B$ overexpression was substantially larger. Whereas the median expression of TOP2A was $31.3 \%$ higher than the median in all other cancer cell lines in DepMap RNA-seq data $\left(P=2 \times 10^{-3}\right.$, Fig. $\left.4 \mathrm{~A}\right)$, the median expression of TOP2B was $87.1 \%$ higher $\left(P=1.3 \times 10^{-7}\right.$, Fig. $\left.4 \mathrm{~B}\right)$. Additionally, TOP $2 B$ expression was among the most highly negatively correlated genes with CX-5461 IC ${ }_{50}$ in GDSC neuroblastoma cell lines $(r=-0.58$, $P=1 \times 10^{-3}$, Fig. $4 \mathrm{C}$ ), and this was the 2 nd highest negative correlation with TOP2B of all drugs screened (Fig. 4D). Based on these observations, we hypothesized that CX-5461's cytotoxicity may occur through preferential targeting of TOP2B, unlike existing TOP2 poisons etoposide and doxorubicin, whose effects primarily result from trapping TOP2A on DNA.

To test this hypothesis, we first performed an shRNA knockdown of TOP2A and TOP2B using five independent shRNAs in CHP-134 and KELLY cells (Fig. 4E and Fig. S4C, D). Remarkably, although knockdown of TOP2A conferred resistance to etoposide, doxorubicin, and CX-5461, knockdown of TOP2B only caused resistance to CX-5461 (Fig. 4F, G, Fig. S4E, $P<0.001)$. The cisplatin-treated negative control was not affected by the knockdown of either gene (Fig. 4F). We next used CRISPRa to overexpress both TOP2A and TOP2B in CHP-134 cells and although overexpression of either gene increased sensitivity to CX-5461, doxorubicin, and etoposide, the effect of TOP2B overexpression was greater for CX-5461 (Fig. $4 \mathrm{H}$, I), corroborating the idea that CX-5461 preferentially interacts with TOP2B.

To confirm direct inhibition of topoisomerase II paralogs by CX-5461, we next performed an in vitro topoisomerase activity assay, which tests a compound's ability to inhibit the decatenation of purified catenated kinetoplast DNA (kDNA) plasmids in the presence of purified topoisomerase protein (Fig. 4J; assay not conducted in neuroblastoma cells). CX-5461 inhibited the activity of both TOP2A and TOP2B-strikingly at concentrations substantially lower than etoposide (Fig. 4K and Fig. S4G, S4J $-\mathrm{M})$. The negative control ciprofloxacin, which targets type II topoisomerase in prokaryotes and is a fluoroquinolone with structural similarity to CX-5461, had no activity in this assay. By comparing the concentration of CX-5461 required to inhibit TOP2A or TOP2B in this highly controlled in vitro system, we directly quantified CX-5461's preference for TOP2B (Fig. S4F). Remarkably, we found that CX-5461 was between 2- and 4-fold more effective at inhibiting TOP2B, compared to TOP2A $(P=2.8$ $\times 10^{-4}$, Fig. $4 \mathrm{~L}$ ), confirming the broader relevance of this mechanism outside neuroblastoma cells. Etoposide and doxorubicin had similar activity against both paralogs. We observed similar trends when we performed a complementary topoisomerase activity assay to determine CX-5461's ability to inhibit topoisomerase II-induced relaxation of purified supercoiled DNA, where CX-5461 performed similarly to doxorubicin and etoposide, but again with a clear preference for TOP2B (Fig. 4M; Fig. S4H). Lastly, to further demonstrate a direct physical interaction between CX-5461 and TOP2B, we performed a photo-crosslinking experiment using a diazirine-derivatized form of CX-5461, whose synthesis was informed by prior structureactivity relationship assays ${ }^{27}$ (see "Methods"). Using this derivative, which also contained an alkyne moiety for click chemistry, we performed biotin-streptavidin purifications of the crosslinked material with biological replicates, showing that CX5461 directly crosslinks to TOP2B in cells (Fig. 4N and Fig S4I). Hence, our findings provide multiple orthogonal lines of evidence demonstrating that CX-5461 exhibits classical TOP2 poison activity, and preferentially targets TOP2B.

Neuroblastoma patient tumors overexpress TOP2B. Given the mechanism responsible for CX-5461's selective killing of neuroblastoma cell lines, we next sought to determine the potential of CX-5461 to treat neuroblastoma patients. We began by using existing patient genomics datasets to interrogate the in vivo activity of the drug target $T O P 2 B$ and to investigate the fidelity of the cell line models used to identify CX-5461. First, it was recently shown that embedding cell lines and patient tumors in a low dimensional latent representation of gene expression data represents a viable strategy to determine whether cancer cell lines recapitulate their tumor-of-origin. We applied this approach ${ }^{37}$ to the DepMap ${ }^{38}$ and GDSC cancer cell lines, which showed that neuroblastoma cell lines were among the most representative of their tumor-of-origin ( $81 \%$ correctly aligned; $P<2.2 \times 10^{-16}$, Fig. 5A, B; Fig. S5A), supporting the relevance of the cell line screening data as a model of neuroblastoma patient tumor biology.

We next explored the in vivo activity of TOP $2 B$ in primary tumors using neuroblastoma patient genomics data. We used an aggregated dataset of 88 diagnostic neuroblastoma tumors where RNA-seq and whole-genome sequencing were available ${ }^{39}$. First, the expression of $T O P 2 B$ in neuroblastoma cell lines was well maintained in patient primary tumors (median expression 104.74 TPM in DepMap neuroblastoma cell lines and 132.4 TPM in our patient tumor cohort; Fig. 5C left). This result was consistent when we normalized against the mean expression of housekeeping genes actin ACTB (ACTIN) and GAPDH to account for dataset-specific effects (Fig. 5C right). We next assessed the expression of TOP $2 B$ across TCGA tumor samples. As was true for cell lines, $T O P 2 B$ was more highly expressed in neuroblastoma tumors than other cancers (Fig. 5D and Fig. S5B, C). We also interrogated expression data from relapsed ${ }^{2}$ and metastatic ${ }^{40}$ neuroblastoma tumors, which had paired primary tumor data and we identified no evidence of depletion of TOP $2 B$ expression in metastases or relapse (Fig. $5 \mathrm{E}-\mathrm{F}$ ). Together, these data are consistent with the high TOP $2 B$ expression in neuroblastoma cell lines being maintained in patient tumors.

Notably, the expression of TOP2B varies over a 14.57 -fold range in these patient tumors $(\min =27.8 \mathrm{TPM}, \max =405.2$ TPM), implying that clinical studies of CX-5461 would benefit from stratifying patients based on the expression of TOP2B. To explore the drivers of these differences, we interrogated the effect of 38 clinical variables and genomic features on TOP2B expression. These included somatic mutations, mutation signatures, copy number aberrations, and structural variants. Because of correlations among these features, we used an ElasticNet regression model $(\alpha=0.5)$ to determine which features were independently predictive of TOP $2 B$ expression. The model identified three predictors: deletion of the $3 \mathrm{p}$ arm of chromosome $3(\operatorname{del}(3 p))$, the $q$ arm of chromosome $11(\operatorname{del}(11 q))$, and patient age-at-diagnosis (Supplementary Data Table S2). TOP2B is on chromosome $3 p$ and as expected, $\operatorname{del}(3 p)$ was associated with a 
a

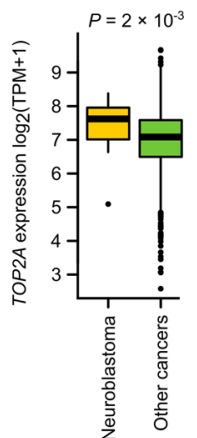

b



C



d

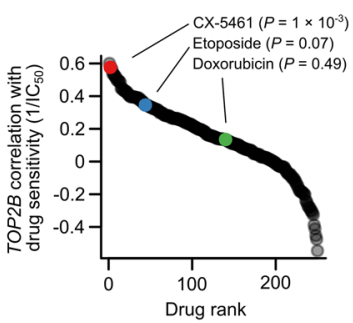

e


\section{f} $\begin{array}{ll}\rightarrow \text { Control shRNA } & \rightarrow-\text { shTOP2B-02560 } \\ \rightarrow \text {-shTOP2A-69079 } & \rightarrow-\text { shTOP2B-55958 } \\ \rightarrow-\text { shTOP2A-86220 } & \rightarrow-\text { shTOP2B-90242 }\end{array}$
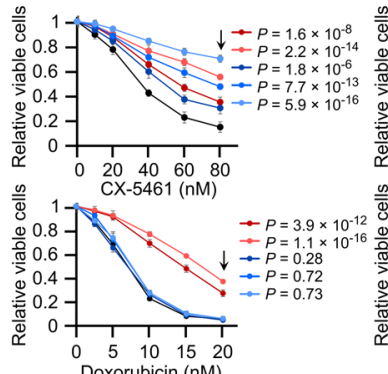

9


j

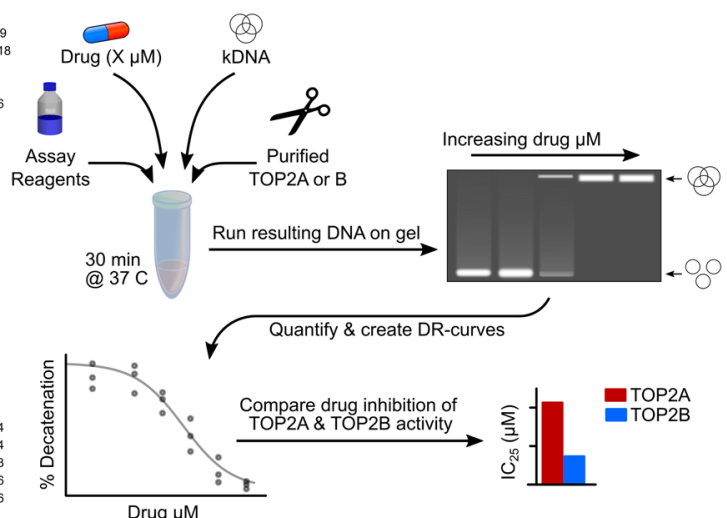

Drug $\mu \mathrm{M}$

$\mathbf{k}$

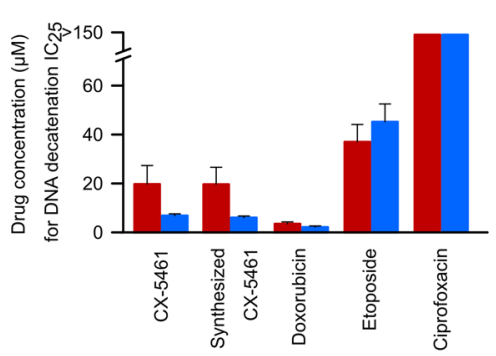

m

Relaxation assay 1


I

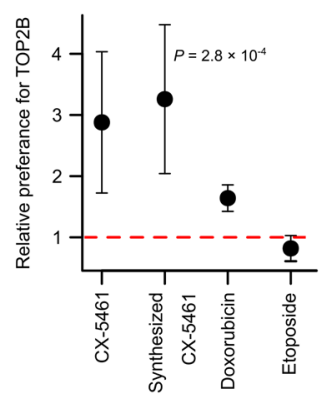

n

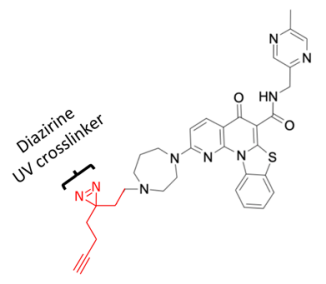

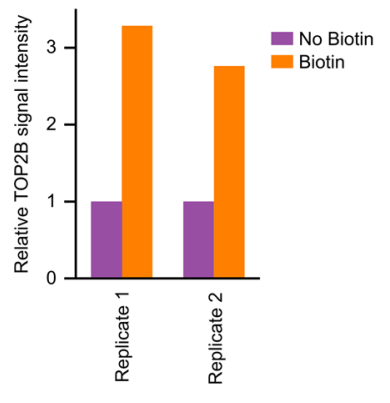

two-fold decrease in its expression (Fig. 5G). Surprisingly, age-atdiagnosis was also a strong predictor of TOP $2 B$ expression, with younger patients expressing much higher levels of TOP2B (Fig. $5 \mathrm{H})$. TOP2B expression was also associated with $M Y C N$ expression when we conditioned on $\operatorname{del}(\mathrm{p} 3)$ and age-at-diagnosis using multivariate linear regression $\left(P=3.3 \times 10^{-3}\right)$. ChIP-seq data also showed that MYCN is bound to the TOP2B promoter (Fig. S5D), consistent with the causal relationship between CX$5461 \mathrm{IC}_{50}$ and $M Y C N$ expression that we observed in cell lines (Fig. 1F). Notably, MYCN and TOP2B expression were also correlated in the cell line data $\left(r=0.59, P=8.1 \times 10^{-4}, n=29\right)$ and both were associated with $\mathrm{CX}-5461 \mathrm{IC}_{50}$. However, when we 
Fig. 4 CX-5461 primarily targets the TOP2B paralog. a The $\log _{2}(T P M+1)$ expression of TOP2A ( $y$-axis) in $n=26$ biologically independent DepMap neuroblastoma cell lines compared to all other $(n=1304)$ DepMap cell lines, measured by RNA-seq. The center line represents the median, bound of box is upper and lower quartiles and whiskers are $1.5 \times$ the interquartile range. $P$-value is from two-sided t-test. b Like (a) but for TOP2B. c Spearman correlations of the expression of all genes with CX-5461 sensitivity in GDSC neuroblastoma cell lines, with TOP2A and TOP2B highlighted. P-values are from two-sided Pearson's correlation tests. $\mathbf{d}$ Spearman correlation of TOP2B expression with sensitivity $\left(1 / \mathrm{IC}_{50}\right)$ of all drugs screened in GDSC neuroblastoma cell lines. CX-5461, etoposide, and doxorubicin are highlighted. P-values are from two-sided Pearson's correlation tests. e TOP2A (left) and TOP2B (right) expression following shRNA knockdown ( 2 shRNAs for TOP2Aand 3 shRNAs for TOP2B). An shRNA with scramble target was used as control. $\beta$-Actin was used as a loading control. Representative results of three biological replicates are shown. $\mathbf{f}$ Cell viability of $\mathrm{CHP}-134$ cells following TOP2A (red) and TOP2B (blue) shRNA knockdown, determined by MTS assay. Data represent mean \pm SD of $n=9$ biological replicates over three independent experiments. $\mathbf{g}$ Viability of TOP2A (red) and TOP2B (blue) knockdown CHP-134 cells treated with $0.05 \mu \mathrm{M}$ or $0.1 \mu \mathrm{M}$ of CX-5461. Data represent mean \pm SD of $n=9$ biological replicates over three independent experiments. h TOP2A (left) and TOP2B (right) expression following CRISPRa (2 gRNAs for each gene) induced overexpression. $\beta$-Actin was used as a loading control. Representative results of three biological replicates are shown. i Viability of TOP2A (red) and TOP2B (blue) overexpressing CHP-134 cells, determined by MTS assay. Data represent mean \pm SD of $n=9$ biological replicates over three independent experiments. $\mathbf{j}$ Schematic of in vitro topoisomerase activity assay (decatenation assay) workflow. $\mathbf{k}$ Drug concentration required to inhibit $25 \%$ of total DNA decatenation $\left(\mathrm{IC}_{25}\right)$. The assay was repeated in $n=5$ independent assays with the error bars representing $95 \%$ confidence intervals (also see Fig. S4f). I Plot showing the relative difference in the concentration of each topoisomerase inhibitor required to inhibit the decatenation of kDNA in the presence of TOP2B relative to TOP2A, calculated from then $=5$ independent assays performed in (k). Error bars represent 95\% confidence intervals. Two-sided test and $P$-values were calculated by simulation. $\mathbf{m}$ Drug concentration required to inhibit $50 \%$ of total DNA relaxation $\left(\mathrm{IC}_{50}\right)$. Two repeat runs are shown in the left and right panels. $\mathbf{n}$ CX-5461-Diazirine structure (left) and quantification of TOP2B protein signal detected in CX-5461-Diazirine treated cells after streptavidin purification (right). Two-tailed student's t-test was used for (f, $\mathbf{g}$, i). Source data for (f, $\mathbf{g}, \mathbf{k}$ $-\mathbf{n})$, are included in Source Data file.

performed mediation analysis, conditioning on the expression of $T O P 2 B$, the association between CX-5461 $\mathrm{IC}_{50}$ and $M Y C N$ expression was lost $(P=0.8)$, but $T O P 2 B$ remained highly significant $\left(P=2.7 \times 10^{-4}\right)$, suggesting that the association between $M Y C N$ and $\mathrm{CX}-5461 \quad \mathrm{IC}_{50}$ was mediated through $M Y C N$ 's effect on TOP $2 B$ expression. Overall, these genomics analyses of patient tumors suggest that high TOP2B activity is maintained and may be therapeutically relevant in a subset of neuroblastoma patients. To explore the potential for a CX-5461 therapeutic window in cancer patients, we also assessed the expression of $T O P 2 B$ in normal human tissues using data from the Genotype-Tissue Expression (GTEx) consortium. Although the expression of $T O P 2 B$ was again typically higher in neuroblastoma tumors than normal human tissues (Fig. 5I), there was clear expression across normal tissues, including cardiac tissues and most strikingly some regions of the brain (Fig. S5E, S5F). TOP2B expression at mRNA and protein levels are also highly consistent (Fig. S5G) ${ }^{41}$. These genomics analyses provide further confidence that CX-5461's selective activity against neuroblastoma cell lines could translate to antineuroblastoma tumor activity in vivo. TOP2B's expression in normal tissues does, however, highlight potential patient safety concerns given the proposed CX-5461 mechanism-of-action.

CX-5461 synergizes with inhibitors of ATM, ATR, and TOP1. In the last decade, single-agent clinical trials in neuroblastoma have almost universally failed, even when the rationale for a new compound has been substantive ${ }^{4}$. For such an aggressive disease, identifying synergistic drug combinations is critical. To address this problem, we leveraged our CRISPR screening data, reasoning that druggable proteins whose genetic knockout synergizes with CX5461 may allow us to narrow the enormous combinatorial search space. For these analyses, we leveraged a previously assembled list of druggable genes ${ }^{12}$. Among the top druggable genes, whose knockout synergizes with CX-5461 in CHP-134 cells were ATM, ATR, and TOP1 (Fig. 6A, B), all genes converging on DNA damage response. Based on our mechanistic work showing the DNA damaging effects of CX-5461 and previous observations of their efficacy in other cancers ${ }^{42,43}$ we chose to investigate drugs targeting these genes as potential combinations with CX-5461. Thus, we screened CHP-134, IMR-5, BE(2)-M17, and KELLY neuroblastoma cell lines with the combination of CX-5461 and AZD-1390 (ATM inhibitor), AZD-6738 (ATR inhibitor), and SN-38 (the active metabolite of irinotecan; TOP1 inhibitor $)^{44-48}$. We screened each of 12 cell line-drug combination pairs with a comprehensive $12 \times 12$ matrix of drug concentrations, achieving this scale using highthroughput robotic handling. We estimated regions of synergy using the Zero Interaction Potency (ZIP) model ${ }^{49}$. Encouragingly, we identified regions of synergy at pharmacologically relevant drug concentrations in all 12 cell line-drug combinations (at a fold change of $>1.5$ relative to the expectation given an additive model, and $P<0.01$, Fig. $6 \mathrm{C}-\mathrm{G}$; Fig. S6A-L). To confirm the validity of the high-throughput screen, we tested several of these combinations using a conventional MTS assay in several cell lines. In every case, the synergy detected by the MTS assay was consistent with the high-throughput screen (Fig. 6H and Fig. S6M). Cell apoptosis and perturbation of ATM/ATR pathways resulting from the drug combinations were also confirmed by western blots and gene expression analysis (Fig. S6N, O). Thus, ATM, ATR, and TOP1 inhibitors represent promising combinations with CX-5461targeted TOP2B inhibition to explore in preclinical in vivo studies.

Establishing a pharmacologically relevant in vivo murine equivalent dosage for CX-5461. Given the selective activity of CX5461 against neuroblastoma cell lines, the rational biological mechanism, and the promising synergistic activity with other compounds in vitro, we sought to perform an in vivo study to test these drug combinations in mice using orthotopic patient-derived xenografts (PDX). To ensure the clinical relevance of our PDX data, we first performed comprehensive in vivo preclinical pharmacokinetics studies, comparing CX-5461 plasma area under the concentration-time curve (AUC) and average plasma concentration (Cavg) values to those reported in the available human phase I clinical trial ${ }^{15}$. This is the first time such a study has been reported for this compound. Notably, our PK results demonstrated highly variable CX-5461 plasma concentrations in mice and revealed a much shorter terminal plasma half-life compared to humans $(83.3 \mathrm{~h}$ in humans at $250 \mathrm{mg} / \mathrm{m}^{2}$ vs $3.7 \mathrm{~h}$ after $5 \mathrm{mg} / \mathrm{kg} \mathrm{IV}$ in mice; Fig. S7A - C; full details in Supplementary Methods). This severely undermines the translational relevance of existing preclinical in vivo CX5461 studies in mice, which have not accounted for these differences. To mitigate these issues we devised a low, multiple-dose CX5461 regimen aimed at maintaining the Cavg above $100 \mu \mathrm{g} / \mathrm{L}$ for 5 days, establishing a dosage of $5 \mathrm{mg} / \mathrm{kg}$ once daily by IP injection for 5 days, starting on day 1 , with a second 5 -day course starting on day 8 , every 21 days. 

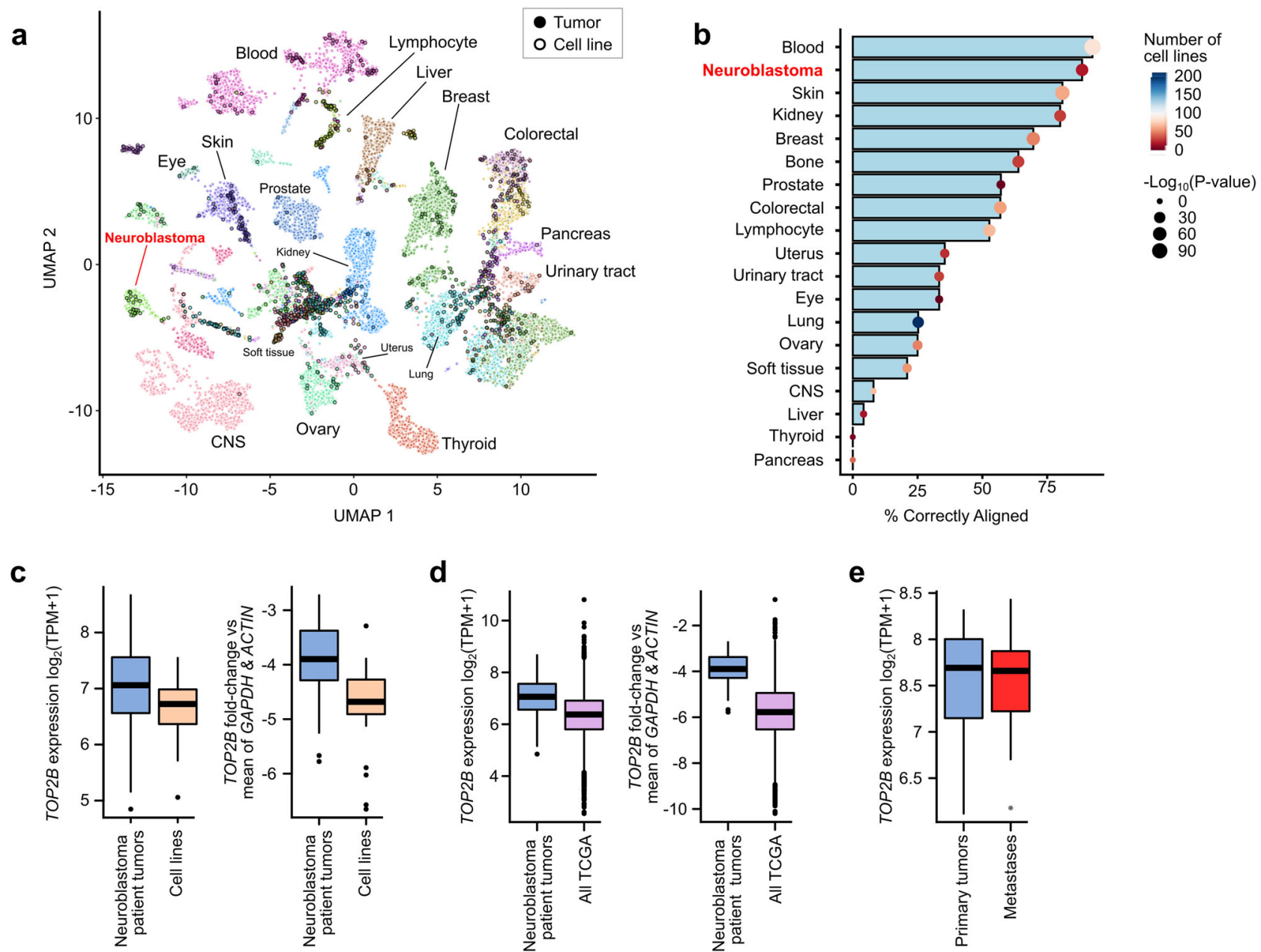

d
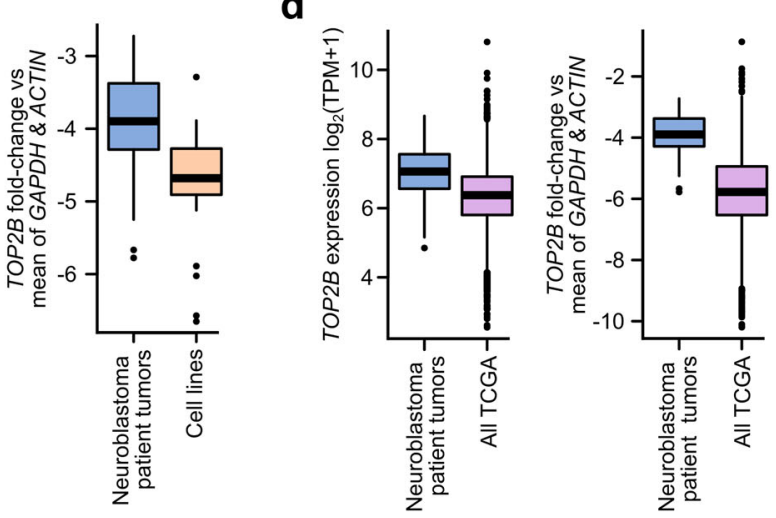

e



f

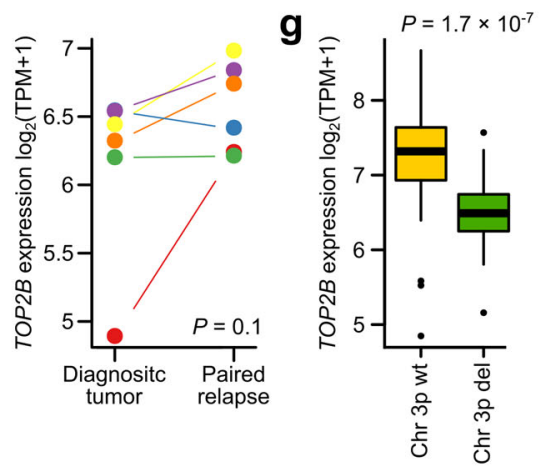

h

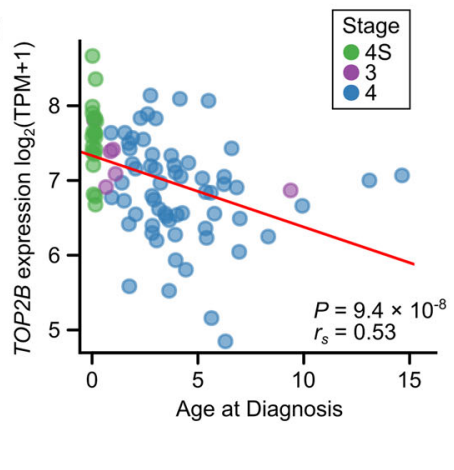





We confirmed on-target drug activity in vivo at these pharmacologically relevant dosages using TOP2cc formation and $\gamma-\mathrm{H} 2 \mathrm{AX}$ as pharmacodynamic markers, interrogated using a RADAR assay (Fig. 7A and Fig. S7D), and immunoblotting respectively (Fig. 7B), using PDX tumor tissue harvested from CX-5461 treated mice. Consistent with our in vitro observations, we observed no inhibition of RNA-POL I, but saw clear TOP2B trapping and DNA damage in these PDX tumors following CX5461 treatment (Fig. 7A-C), confirming the in vivo relevance of our mechanistic work.

CX-5461 improves survival in vivo when combined with TOP1 inhibitors in orthotopic PDX mouse models. We performed our in vivo drug treatments using two luciferase-labeled PDXs-
SJNBL046, a MYCN amplified model from a 2-year-old male, and SJNBL047443, an ATRX mutant model from a 12-year-old male. Both PDXs were from pre-treated neuroblastoma tumors, with SJNBL046 surgically resected after induction chemotherapy and SJNBL047443 resected at disease recurrence. We used RNA-seq to compare genome-wide gene expression in these PDXs to their patient tumor of origin and the expression profiles were well maintained $\left(r=0.91, P<2.2 \times 10^{-16}\right.$ for SJNBL046; $r=0.75$, $P<2.2 \times 10^{-16}$ for SJNBL047443; Fig. 7D, E); TOP2B expression was also maintained, suggesting these PDXs are a relevant disease model. We implanted the cells by ultrasound-guided injection into the orthotopic para-adrenal region of athymic-nude mice, and we monitored tumors using bioluminescent imaging and weekly ultrasound (Fig. 7F, G; Fig. S7E, F). 
Fig. 5 TOP2B expression is similar in neuroblastoma patient tumors and cell lines, but it is also expressed in normal cells. a UMAP representation showing the genome-wide gene-expression-based alignment of all DepMap cell lines with patient tumor gene expression data from the UCSC Xena repository [xenabrowser.net] encompassing TCGA, Treehouse, and TARGET datasets. Alignment was generated using the Celligner tool. The points representing individual cell lines have a black border. Points have been colored by their lineage and clusters have been labeled by tumor lineage. $\mathbf{b}$ The proportion of cell lines from a given lineage that correctly align with the appropriate patient tumor cluster ( $x$-axis). The points have been colored by the number of cell lines in the datasets. The size of the points has been scaled by the $P$-value obtained from a Fisher's exact test for cluster membership. c Boxplot of TOP2B expression in $n=26$ biologically independent neuroblastoma cell lines obtained from DepMap RNA-seq vs 88 biologically independent neuroblastoma patient tumors for $\log _{2}(T P M+1)$ normalized RNA-seq expression (left) and TOP2B expression in these datasets normalized to $A C T I N$ and GAPDH housekeeping genes (right panel). d TOP2B RNA-seq expression data in 88 neuroblastoma patient tumors compared to all $n=9,968$ biologically independent TCGA tumors for $\log _{2}(T P M+1)$ normalized expression values (left) and TOP2B normalized to ACTIN and GAPDH housekeeping genes (right). e TOP2B $\log _{2}(T P M+1)$ normalized expression at $n=6$ biologically independent primary sites and metastatic sites in published RNA-seq data obtained from Rifatbegovic et al. ${ }^{40}$. f TOP2B $\log _{2}(T P M+1)$ normalized RNA-seq expression obtained from six patients in the TARGET cohort for whom both diagnostic and matched relapse tumor gene expression data were available. $\mathbf{g}$ Boxplot showing TOP2B $\log _{2}($ TPM +1$)$ normalized RNA-seq expression from $n=88$ biologically independent neuroblastoma patient tumors with a chromosome $3 p$ deletion $(n=25)$ compared to the wild-type (wt) copy ( $n=63)$ of chromosome $3 p$. $\mathbf{h}$ Scatterplot of TOP2B $\log _{2}(T P M+1)$ normalized RNA-seq expression ( $y$-axis) plotted against age ( $x$-axis) for 88 neuroblastoma patient tumors. Patients are colored by disease stage, with stage 4S, 3 and 4 patients colored green, purple, and blue respectively. i TOP2B RNA-seq expression data in 88 neuroblastoma patient tumors compared to all GTEx normal tissues for $\log _{2}(T P M+1)$ normalized expression values (left) and TOP2B normalized to ACTIN and GAPDH housekeeping genes as negative controls (right). GTEx data include $n=57$ tissues type obtained from $n=948$ biologically independent donors. In all boxplots, the center line represents the median, bound of box is upper and lower quartiles and the whiskers are $1.5 \times$ the interquartile range.

We first tested 2-drug combinations of CX-5461 with pharmacologically relevant dosages of AZD-1390 (ATMi), AZD-6738 (ATRi), and the liposomal formulation of the TOP1 inhibitor irinotecan. We also tested 3-drug combinations involving CX-5461, irinotecan, and the ATMi or ATRi (Fig. 7H). Combinations involving CX-5461 + ATMi had clear toxicity and were associated with lower body weight (Fig. 7I; Fig. S7G). Indeed, for the CX-5461 + ATMi two-drug combination, 30\% (MYCN amplified group) and 36.8\% (ATRX mutant group) of mice were withdrawn (censored) from the study, primarily due to toxicity; this increased to $81.8 \%$ and $73.7 \%$ with the addition of irinotecan (Fig. 7J, Supplementary Data Tables S3, S4). The combinations involving ATRi lacked efficacy in vivo, with the two-drug combination of CX-5461 + ATRi showing no activity beyond placebo, and no increase in efficacy was observed with the addition of ATRi to CX-5461 + irinotecan (Fig. 7J). However, the dual treatment of CX-5461 with the TOP1 inhibitor irinotecan was effective, resulting in improved outcomes relative to placebo for both PDXs $\left(P=0.03\right.$ and $P=9.4 \times 10^{-5}$ for MYCN and ATRX PDXs respectively; Supplementary Data Tables S5, S6). Importantly, no mice were censored due to toxicity.

We designed a second study to further explore the combination of CX-5461 with TOP1 inhibitors, combining CX-5461 with two 2-drug salvage combinations used in relapsed and refractory neuroblastoma, which have been used as backbones for incorporating new agents in clinical trials: irinotecan + temozolomide and topotecan + cyclophosphamide (Fig. 7K; Supplementary Data Tables S7, S8) ${ }^{50,51}$. The therapies were reasonably tolerated with minimal loss of body weight (Fig. 7L; Fig. S7H) or censoring (Fig. 7M). For both regimens, the addition of CX-5461 improved outcome in every group in both PDXs, clearest in the irinotecan + temozolomide combination in the SJNBL047443 PDXs $(P=0.05$; Supplementary Data Tables S9, S10). Thus, in all six independent experiments, CX-5461 improved survival when added to a TOP1 inhibitor $(P=0.01)$. The CX-5461 treated mice also survived longer following cessation of therapy in all groups (mean $71-83.75$ days and 90.8-144 days for the addition of CX-5461 to cyclophosphamide and topotecan, $21.75-24.8$ days and 4-18.8 days for the addition of CX-5461 to irinotecan and temozolomide for the MYCN and ATRX mutant PDX groups respectively; Supplementary Data Tables S7, S8).

Overall, our mouse studies suggest that combinations of CX5461 with TOP1 inhibitors are synergistic in vivo and have clinical potential in neuroblastoma. However, despite the antitumor activity, our mechanistic work raises the possibility of lateemerging adverse events when TOP2B is targeted, which needs to be further understood before this combination is applied in a pediatric patient cohort.

\section{Discussion}

We used large-scale preclinical drug screening data to identify CX-5461 as exhibiting a selective and reproducible cytotoxic effect in high-risk neuroblastoma and we discovered that the primary target of CX-5461 at pharmacologically relevant concentrations is TOP2B. Because of the functionally unique role of TOP2B, this finding has potential clinical implications for CX5461 in terms of patient stratification, rational combinations, potential resistance mechanisms, and safety profile.

Evidence that CX-5461 inhibits the transcription of rRNA has fueled previous conclusions that CX-5461 directly targets RNAPOL I or the machinery associated with RNA-POL I activity machinery ${ }^{52,53}$. Similarly to other topoisomerase inhibitors ${ }^{54-57}$, several studies have implicated CX-5461 as a binder of DNA G-quadruplexes in vitro ${ }^{57-59}$, and it has been argued that CX5461 targets TOP2 $\mathrm{A}^{33}$ (TOP2B was not mentioned in ref. ${ }^{33}$ ); this was subsequently speculated to relate to G-quadruplexes ${ }^{60}$. However, convincing direct evidence of these mechanisms has been lacking and none of these previously proposed mechanisms of CX-5461 unified the wide range of observations made on this compound-nor unambiguously identified its molecular target.

The activity of CX-5461 primarily as a TOP2B inhibitor is consistent with numerous previous observations, including CX5461's DNA damaging effects ${ }^{59}$ and its tendency to inhibit RNAPOL I-mediated rRNA transcription ${ }^{21}$ and nucleolar integrity 61,62 , even in non-cycling cells that do not express TOP $2 A^{35}$. Critically, multiple previous studies have shown that topoisomerases play a key role in resolving DNA topological issues to maintain active transcription ${ }^{63}$, including at the rRNA loci ${ }^{64}$ and knockdown of topoisomerases, as well as treatment with topoisomerase inhibitors, has been established to cause inhibition of RNA-POL ${ }^{65,66}$ (shown again here in Fig. S4D). Additionally, TOP2B has been shown to localize to the rRNA locus, where RNA-POL I activity occurs ${ }^{67}$. Thus, our study leads us to a parsimonious model, able to explain the capabilities of CX-5461 to induce DNA damage ${ }^{59,68}$ and inhibit rRNA transcription. We specifically propose that the primary molecular 

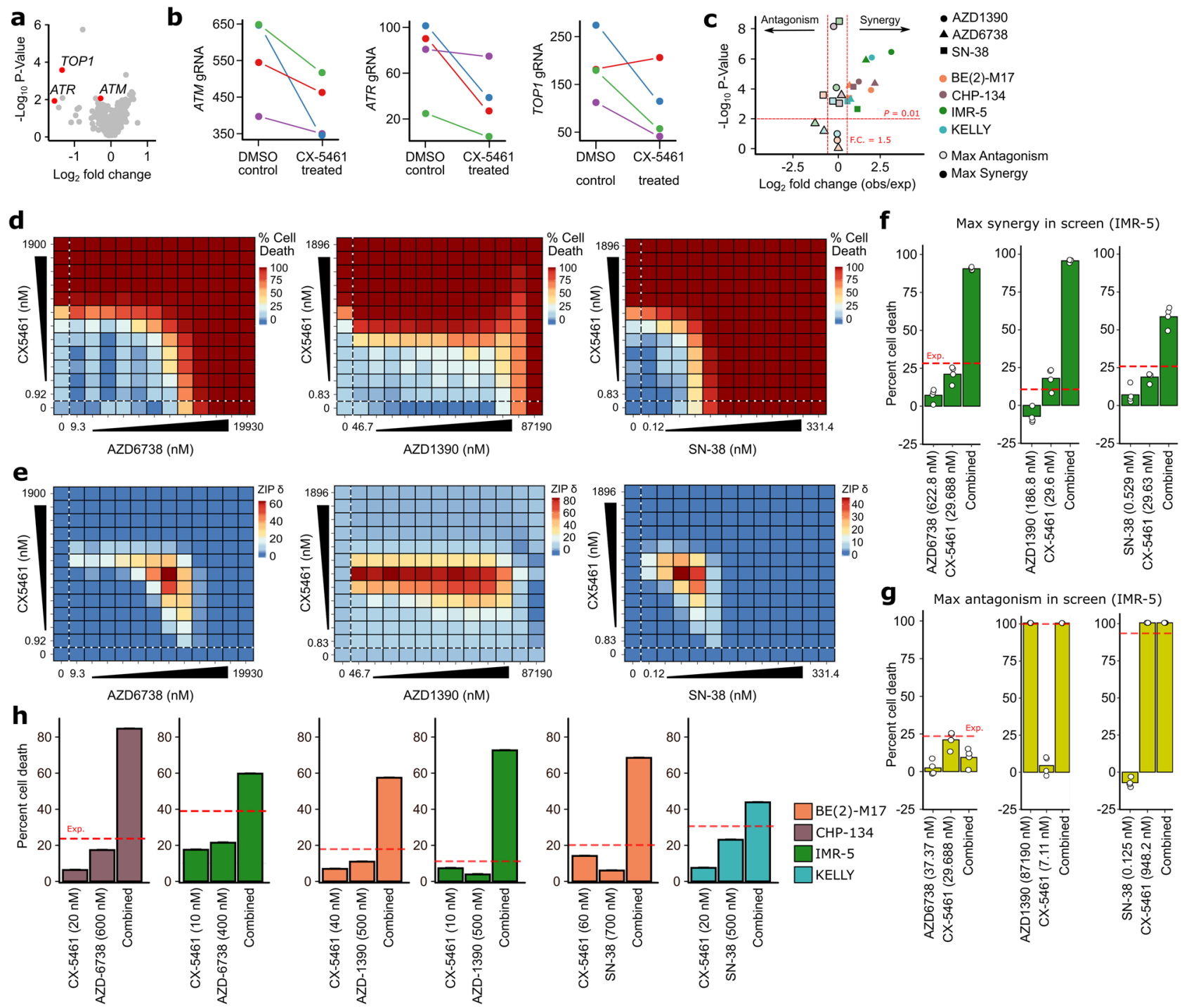

SN-38 (nM)
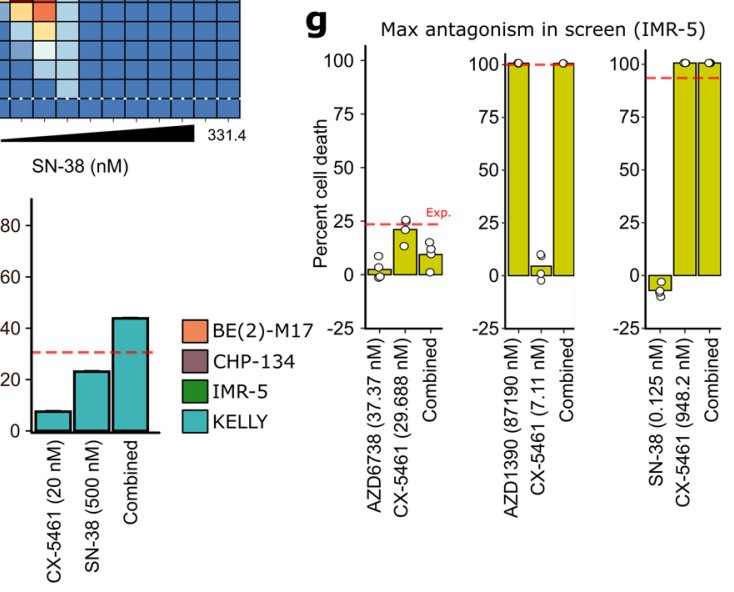

Fig. 6 CX-5461 synergizes with inhibitors of ATM, ATR, and TOP1. a Sensitization of CHP-134 cells to CX-5461 following CRISPR knockout of known drug target genes (using a modified version of the list previously assembled by Behan et al., ${ }^{81}$ ). b Normalized guide-RNA (gRNA) read counts ( $y$-axis) for each of four independent guides used to knockout ATM (left), ATR (middle), and TOP1 (right). c Volcano plot depicting fold-changes ( $x$-axis) and P-values ( $y$-axis) at maximum synergy (unbordered shapes) and maximum antagonism (bordered shapes) scores observed in all 12 drug combination screening assays. The shape indicates which drug was combined with CX-5461 and the color of each point indicates the cell line screened. Fold-change is calculated relative to the expectation given additive drug activity and $P$-values were calculated using a one-sample t-test. Thresholds correspond to absolute log ${ }_{2}$ (fold change) $\geq 1.5$ and $P$-value $\leq$.01. d Heatmap matrices of percent cell death in IMR- 5 cells conferred by drug-only treatments (values within dotted lines) and combination treatments (all other values) for CX-5461 with AZD6738 (left), AZD1390 (middle), and SN-38 (right). Twelve-point doses were used at indicated concentrations with 1:2-fold dilutions for all compounds. Each matrix represents the average of four independent experiments. Data were normalized as a function of percent cell death according to inter-plate controls. e Heatmap matrices of synergy scores derived from respective cell death values in (d). All synergy was calculated based on the zero interaction potency (ZIP) model, and corresponding scores are shown as $\delta$ ZIP according to the original method. Combinations conferring synergy or antagonism correspond to values greater than or less than zero, respectively. Bar plots of drug combinations that confer the maximum synergy (f) or maximum antagonism ( $\mathbf{g}$ ) scores from their respective synergy calculations. White dots represent four independent experiments corresponding to score maxima. Red dotted lines represent the expected result based on additivity alone. $\mathbf{h}$ Synergy validation with MTS assays for CX-5461 in combination with AZD6738, AZD1390, or SN-38. Colors correspond to cell lines. Data are representative of three independent experiments. The source data are included in Source Data file.

target of CX-5461 is TOP2B, and that previously observed inhibitory effects of CX-5461 on RNA-POL I are a consequence of topoisomerase inhibition.

Interestingly $T O P 2 B$ knockout mice die shortly after birth due to defects in the peripheral nervous system occurring during the late stages of development ${ }^{69}$, suggesting TOP2B's relevance as a drug target in neuroblastoma partially stems from the tumor's developmental cell-of-origin. We showed that TOP2B remains overexpressed in neuroblastoma, particularly in younger and neonatal patients and that $T O P 2 B$ expression is driven higher by $M Y C N$ overexpression, explaining the selective activity of CX-5461 against neuroblastoma cells. However, while we showed anti-tumor activity in combination with TOP1 inhibitors in vivo in orthotopic PDX models at pharmacologically relevant dosages, the clinical translation of CX-5461 in neuroblastoma is likely to face an unexpected obstacle-as CX-5461's preferential targeting of TOP2B raises major previously unanticipated safety concerns. Existing TOP2A inhibitors can cause serious late-emerging side effects, which would 
a

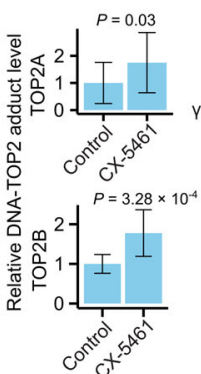

b CX-5461 delivery by



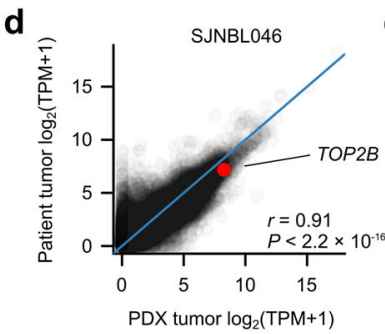

Week 3
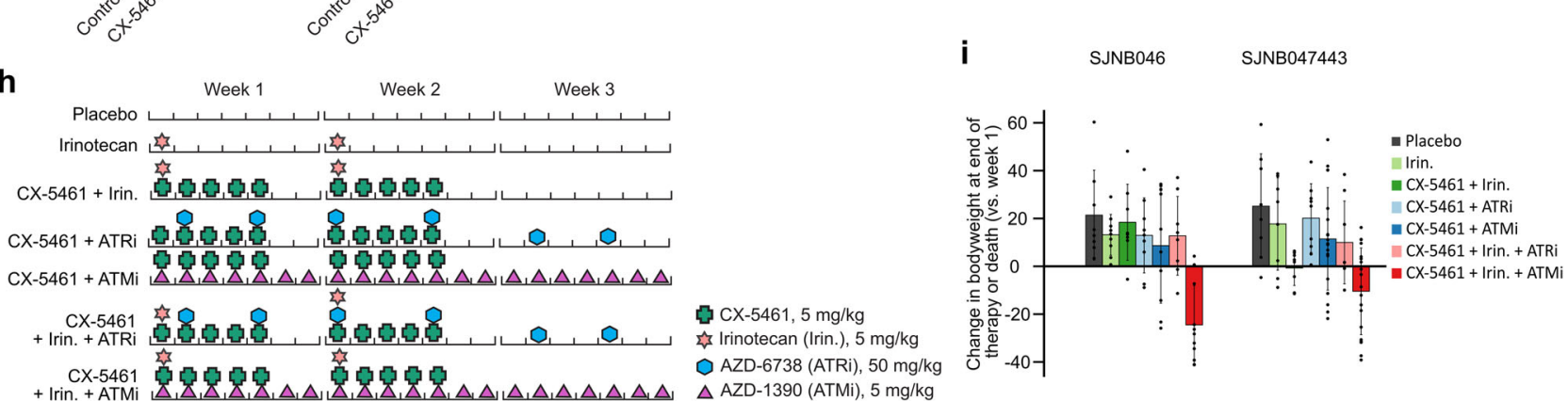

j
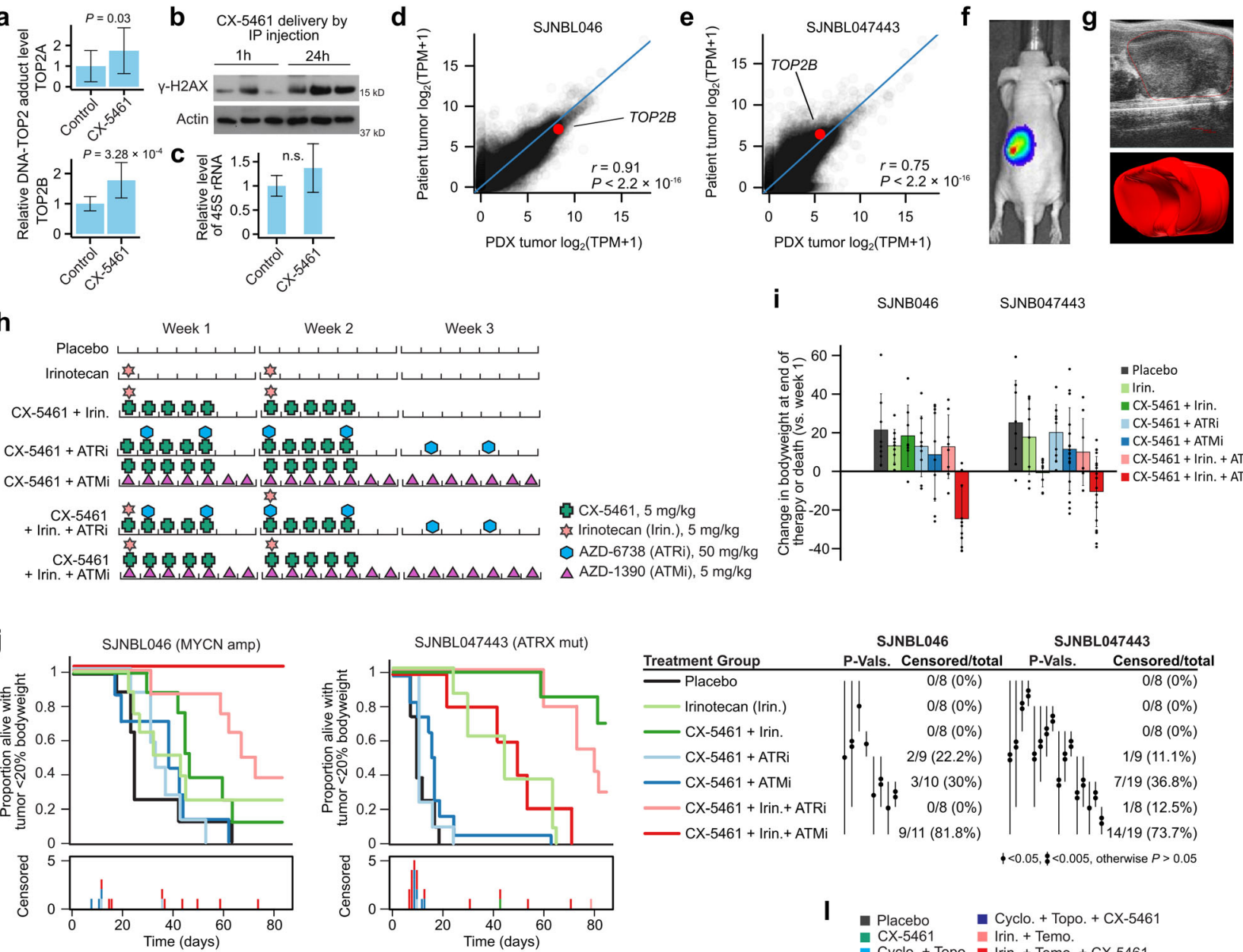

+ Irin + ATMi

$\mathbf{k}$

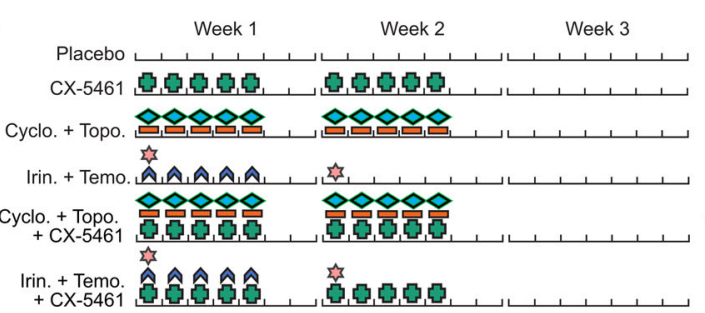

CX-5461, $5 \mathrm{mg} / \mathrm{kg}$ $\checkmark$ Cyclophosphamide (Cyclo.), $50 \mathrm{mg} / \mathrm{kg}$ - Topotecan (Topo.), $0.3125 \mathrm{mg} / \mathrm{kg}$ Irinotecan (Irin.), $5 \mathrm{mg} / \mathrm{kg}$
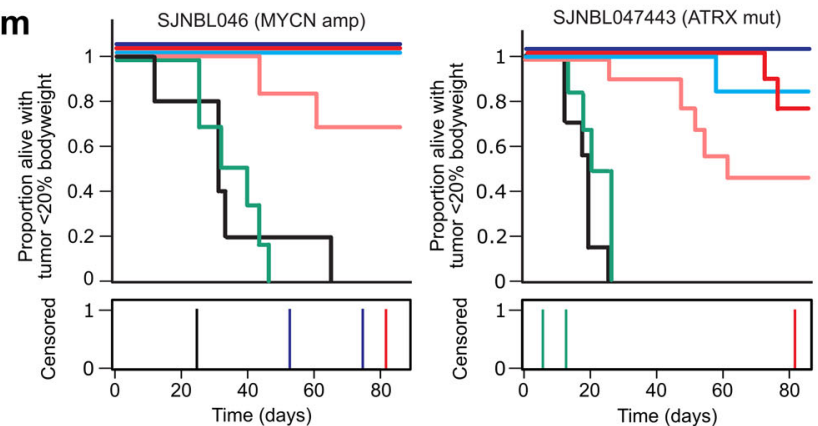

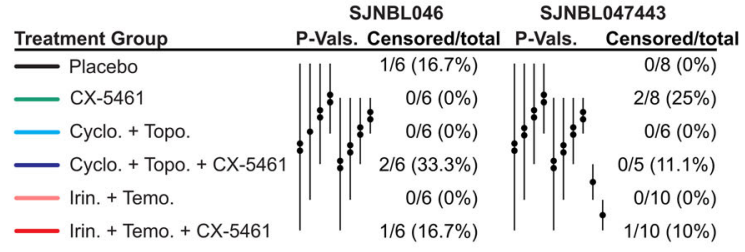

$\phi<0.05,<0.005$, otherwise $P>0.05$ not be observable in a typical preclinical study, nor in shortduration phase I clinical trials. For example, etoposide leads to therapy-induced AML 2-3 years after treatment in about $1 \%$ of patients, and doxorubicin causes (frequently fatal) cardiotoxicity in about $11 \%$ of patients, both of which are caused by off-target interactions with TOP2B ${ }^{17,18}$. Consistent with this, our analysis of GTEx data showed that TOP2B is broadly and highly expressed in normal human tissues, which is not true of TOP2A. CX-5461 has recently been shown to be mutagenic in a $C$. elegans model ${ }^{70}$, contradicting earlier claims that it is not genotoxic ${ }^{21}$. Thus, our finding that CX-5461 primarily targets TOP2B provokes serious safety concerns, and patients enrolled in ongoing CX-5461 phase II trials should be closely monitored for these late-emerging $T O P 2 B$ related adverse events. TOP $2 B$ is also particularly highly expressed in the brain, suggesting that blood-brain barrier penetration should be formally assessed. 
Fig. 7 CX-5461 shows promising anti-tumor activity in combination with TOP1 inhibitors in vivo using orthotopic PDX mouse models. a Quantification of RADAR assay for TOP2-DNA adduct formation in SJNBL046 PDXs harvested from CX-5461 treated mice or control. Tissues were obtained from our placebo and CX-5461 group in Fig. 7m. Data represent the mean \pm SD of 12 replicates. b Western blots for $\gamma-H 2 A X$ in tumor tissue obtained during PK study. Mice were treated with $25 \mathrm{mg} / \mathrm{kg}$ CX-5461 by IP injection and tumors harvested at $1 \mathrm{~h}$ or $24 \mathrm{~h}$. $\beta$-Actin was used as a loading control. c RT-qPCR for 45 s rRNA in the same tissue as Fig. 7A. Data represent the mean \pm SD of 12 replicates. n.s. no significant difference. $\mathbf{d}$ Genome-wide gene expression as measured by RNA-seq in MYCN amplified patient tumor SJNBL046 and in the PDX derived from this tumor. TOP2B is highlighted. e Same as (d) but showing data from the ATRX mutant sample SJNBL047443. Representative image from tumor monitoring using luciferase bioluminescence imaging (f) and ultrasound $(\mathbf{g})$. h Drug combination schedule depicted for a single cycle in study 1 . Mice were treated for four cycles. $\mathbf{i}$ Change of bodyweight (\% of week 1) for each group of mice at the end of therapy or at death (the mice that died before the completion of therapy) in study 1 (described in (h)). Each datapoint represents a mouse. Data represent the mean \pm SD. P-values (left to right): SJNB046, $9.2 \times 10^{-3}, 2.4 \times 10^{-3}, 7.4 \times 10^{-3}, 0.043,0.29,0.078,2.3$ $\times 10^{-4}$; SJNB047443, 0.11, 0.027, 0.88, $3.4 \times 10^{-3}, 0.036,0.15,0.030 . \mathbf{j}$ Kaplan-Meier curves showing the proportion of mice alive in each treatment group with a tumor burden estimated at $<20 \%$ of bodyweight in study 1 (described in (h)). The number of mice censored in each treatment group at each time point is shown below. $P$-values were calculated between pairs of curves using a log-rank test and are depicted by a vertical line, with the number of dots corresponding to the level of significance; comparisons yielding $P>0.05$ are not shown. $\mathbf{k}$ Drug combination schedule depicted for a single cycle in study 2. Mice were treated for four cycles. I Like (i) but showing bodyweights for study 2 (described in (k)). Each datapoint represents a mouse. Data represent the mean \pm SD. $P$-values (left to right): SJNB046, 0.020, $2.3 \times 10^{-3}, 0.11,0.99,0.44,0.44$; SJNB047443, 1.2 × 10-3, 1.3 × 10-5, 0.020, 0.80, $0.73,0.65$. $\mathbf{m}$ Like (j) but showing the results of study 2 (described in ( $\mathbf{k})$ ). Two-tailed t-test was used for $(\mathbf{a}, \mathbf{c}, \mathbf{i}, \mathbf{I})$. Source data for $\mathbf{a}, \mathbf{c}, \mathbf{i}, \mathbf{j}, \mathbf{I}$, and $\mathbf{m}$ are included in Source Data file.

Our analysis of TCGA data showed that subsets of both breast and hematological cancer patients (the diseases of ongoing trials) also overexpress $T O P 2 B$, most strikingly in some leukemias. Our identification of CX-5461's primary target will facilitate patient stratification in these trials, which along with rational drug combinations will markedly improve the chances of successful phase II trials in adult cancers, if safety concerns can be addressed. Indeed, given the concerns surrounding the safety of TOP2B as a primary drug target, achieving promising results in an adult cohort will likely be a prerequisite for a clinical study in children, where the barriers to new clinical trials are much higher due to the limited and highly vulnerable patient population.

\begin{abstract}
Methods
Cell lines and cell culture conditions. CHP-134, KELLY, and SK-N-SH cells were purchased from Sigma (MilliporeSigma, USA). BE(2)-M17, SK-N_FI, RS4-11, H2452, and 293T cells were purchased from ATCC. IMR-5 cell line was a gift from the Cellular Screening Center at the University of Chicago. IMR- 5 was cultured in Eagle's Minimum Essential Medium (EMEM, ATCC catalog\# 30-2003) supplemented with fetal bovine serum (FBS, ATCC $30-2020^{\mathrm{mm}}$ ) to a final concentration of $10 \%$. The rest of the cell lines were cultured under conditions recommended by the vendors. All cells are cultured at $37^{\circ} \mathrm{C}$ with $5 \% \mathrm{CO}_{2}$. No cell lines used in this study were found in the database of commonly misidentified cell lines that are maintained by ICLAC and NCBI Biosample. Cell lines were tested negative for mycoplasma using the MycoAlert mycoplasma detection kit (Lonza, LT07-118).
\end{abstract}

Transfection and transduction. Inducible TRIPZ lentiviral shRNA of human TOP2A, TOP2B, and negative control shRNA plasmids were purchased from Dharmacon (Horizon Discovery, USA. TOP2A shRNA clone numbers are RHS4696-200686220, RHS4696-200769079. TOP2B clone numbers are RHS4696200690242, RHS4696-200702560, RHS4696-200755958). MYCN shRNAs were ordered from Transomic Technologies (Cat\# TLHSU2300-4613. Clone numbers are ULTRA-3327035, ULTRA-3327037, ULTRA-3475807). Lentiviral envelope and packing plasmids pMD2.G and psPAX2 were ordered from Addgene (pMD2.G \#12259, psPAX2 \#12260). To generate lentiviral particles, 293T cells were seeded in a six-well plate one day before transfection. For one well of a six-well plate, cells were co-transfected with $0.56 \mu \mathrm{g}$ pMD2.G, $0.83 \mu \mathrm{g}$ psPAX2, and $1.1 \mu \mathrm{g}$ shRNA plasmid, using Lipofectamine 3000 (ThermoFisher, USA. Catalog\# L3000008). Cell culture medium with lentivirus was collected three times during the time period 36 to $72 \mathrm{~h}$ after transfection. CHP-134 cells were infected two to three times during $24 \mathrm{~h}$. Two days after infection, $0.5 \mu \mathrm{g} / \mathrm{ml}$ puromycin was added to the culture medium to select infected positive cells. $2 \mu \mathrm{g} / \mathrm{ml}$ doxycycline was used to induce shRNA expression. For MYCN knockdown, Cells were transduced with three independent $M Y C N$ shRNAs or a negative control shRNA. After 6 days of incubation in a medium with $2 \mu \mathrm{g} / \mathrm{ml}$ doxycycline, cells were treated with CX-5461 for 3 days. For TOP2A and TOP2B knockdown, CHP-134 cells were transduced with TOP2A or TOP2B shRNAs (2 shRNAs for TOP2A and 3 shRNAs for TOP2B). A control shRNA was used with scramble target in human cells. Cells were cultured in medium with $0.5 \mu \mathrm{g} / \mathrm{ml}$ puromycin and $2 \mu \mathrm{g} / \mathrm{ml}$ doxycycline for 3 days, then cells were collected for western blotting or treated with drugs for $72 \mathrm{~h}$ for cell viability assay.
CRISPR activation sgRNA plasmids for TOP2A (Cat\# GSGH11887-247255376, GSGH11887-247255378) and TOP2B (Cat\# GSGH11887-247255386, GSGH11887-247255388), and plasmids for dCas9 expression (Cat\# CAS11914) were ordered from Horizon Discovery. Lentiviral particles were generated with cotransfection with pMD2.G and psPAX2 in $293 \mathrm{~T}$ cells. CHP-134 cells were infected with dCas9 lentivirus and dCas9-expressing CHP-134 cells were selected with $7 \mu \mathrm{g} /$ $\mathrm{ml}$ blasticidin. The virus with TOP2A or TOP2B CRISPR activation sgRNAs was used to infect the blasticidin-resistant cells. Positive cells with TOP2A or TOP2B sgRNAs were selected with $0.5 \mu \mathrm{g} / \mathrm{ml}$ puromycin.

RNA extraction and quantitative real-time RT-PCR. Total RNA was extracted using Trizol (Invitrogen) according to the manufacturer's protocol. cDNA was synthesized using qScript cDNA Synthesis Kit (Quanta Biosciences, catalog\# 95047). SYBR Green PCR Master Mix (Quanta Biosciences, catalog\# 95072-012) was used for the quantitative real-time RT-PCR, which was performed on the CFX96 real-time PCR detection system (Biorad). Experimental results were normalized to $\beta$-Actin. The primers for $45 S$ pre-rRNA internal transcribed spacer region and 18s rRNA were described in ref. ${ }^{58}$. 28S-rRNA primers were described in ref. ${ }^{22}$. All primer sequences are listed in Supplementary Data Table S11.

Flow cytometry. For apoptosis, cells were treated in six-well plates. After incubation for the time needed, the cell culture medium and cells were collected and washed in cold phosphate-buffered saline (PBS). The cells were then resuspended in annexin-binding buffer and stained with Alexa Fluor 488 conjugated annexin V (Thermo Fisher, catalog\# A13201) and $1 \mu \mathrm{g} / \mathrm{ml}$ Propidium iodide (PI) for flow cytometry analysis. For cell cycle analysis, cells were fixed with cold $70 \%$ ethanol in water. After $2 \times$ washes in PBS, cells were treated with $100 \mu \mathrm{g} / \mathrm{ml}$ RNase A and stained with $50 \mu \mathrm{g} / \mathrm{ml}$ PI for flow cytometry analysis. For $\gamma$-H2AX staining, cells were fixed with $4 \%$ formaldehyde and permeabilized with $90 \%$ methanol, then stained with $\gamma$-H2AX (Cell Signaling, \#9718) and Alexa fluor 594 conjugate antirabbit IgG (Cell Signaling, 8889) for flow cytometry. All samples were analyzed with BD LSRFortess flow cytometer. Data were collected and analyzed with BD FACSDiva and FlowJo software. Gating strategies for flow cytometry are shown in Fig. S8.

MTS and CellTiter-Glo assays. Cells were seeded in 96-well plates and treated with compounds as needed. Premixed MTS reagent (Abcam, ab197010) was added to the medium in a 96-well plate to a final concentration of $10 \%$. Then the clear bottom plate was incubated in a cell culture incubator for $0.5-4 \mathrm{~h}$ and absorbance was measured at $490 \mathrm{~nm}$. For CellTiter-Glo (Promega, G9241), the reagent was added to the white bottom plate to a final concentration of $50 \%$. The plate was then shaken for $2 \mathrm{~min}$ to induce cell lysis. After incubation at room temperature for $10 \mathrm{~min}$, the luminescence was recorded.

Western blotting and band depletion assay. Generally, cells were washed with cold PBS and lysed in RIPA buffer (1\% sodium deoxycholate, $0.1 \%$ SDS, $1 \%$ Triton $\mathrm{X}-100,10 \mathrm{mM}$ Tris [pH 8.0], and $150 \mathrm{mM} \mathrm{NaCl}$ ) in cell culture plates. RIPA buffer was supplemented with Protease Inhibitor Cocktail (Sigma, \#5892970001) and Phosphatase Inhibitor (Thermo Fisher, \#A32957). For cleaved PARP detection, cell culture medium was also collected. Protein concentration was determined by BCA protein assay kit (Thermo Fisher, catalog\# 23225). After boiled in 1× LDS sample buffer (Thermo Fisher, \#NP0007), proteins were separated by SDS-PAGE and transferred to PVDF membranes. After blocking in 5\% non-fat milk in TBS with 
$0.1 \%$ Tween-20, membranes were probed with primary antibodies and horseradish-peroxidase-conjugated secondary antibodies. The signal was visualized by Western Lightning Plus-ECL (PerkinElmer). Band depletion assay was performed as previously described with slight modification ${ }^{30}$. Cells were exposed to agents at their approximate IC50 for $4 \mathrm{~h}$. Treated cells were lysed in culture plates with $1 \times$ Laemmli sample buffer ( $2 \%$ SDS, $10 \%$ glycerol, $0.002 \%$ bromphenol blue, $0.0625 \mathrm{M}$ Tris- $\mathrm{Cl}$ (pH 6.8), 10\% 2-mercaptoethanol). Samples were boiled and $10 \mu \mathrm{l}$ of each sample was separated by SDS-PAGE. Cell lysates from untreated cells were used as loading standard. For lanes $1-4$, protein equal to $33,16.5,8.3$, and 4.1 thousand cells were loaded respectively. For each of the rest of the lanes, protein equal to 33 thousand cells was loaded. The antibodies used for western blotting in this study are $\beta$-Actin (Sigma, \#A1978; 1:20,000 dilution), cleaved PARP (Cells Signaling, \#5625; 1:1,000 dilution), TOP1 (Santa Cruz, \#sc-271285; 1:200 dilution), TOP2A (Santa Cruz, \#sc-365916; 1:200 dilution), TOP2B (BD Biosciences, \#611492; 1:2,000 dilution), P53 (Santa Cruz, \#sc-126; 1:200 dilution), phospho-P53 S15 (Cell signaling, \#9284; 1:1,000 dilution), pCHK1 S345 (Cell Signaling, \#2348; 1:1,000 dilution), CHK1 (Santa Cruz, \#sc-8408; 1:200 dilution), pCHK2 T68 (Cell Signaling, \#2661; 1:1,000 dilution), CHK2 (Santa Cruz, \#sc-17747; 1:100 dilution), HRP-linked anti-rabbit IgG (Cell Signaling, \#7074; 1:2,000 dilution), HRP-linked anti-mouse IgG (Cell Signaling, \#7076; 1:2,000 dilution). All unprocessed images are included in Fig. S9.

Immunofluorescence, EU and EdU incorporation. Cells were seeded on poly-Dlysine coated coverslips before treatment. Treated cells were fixed in $4 \%$ formaldehyde for $15-20 \mathrm{~min}$ and permeabilized with $0.3 \%$ Triton X-100 for $10 \mathrm{~min}$ at room temperature. For $\gamma$-H2AX, 53BP1, and RPA staining, cells were blocked in $5 \% \mathrm{FBS} / \mathrm{PBS}$ for $1 \mathrm{~h}$ at room temperature, then incubated with primary antibody diluted in $1 \% \mathrm{FBS} / \mathrm{PBS}$ overnight at $4{ }^{\circ} \mathrm{C}$. Fluorochrome-conjugated secondary antibodies were incubated for $1 \mathrm{~h}$ at room temperature. ProLong ${ }^{\oplus}$ Gold Antifade Reagent with DAPI (Cell Signaling, \#8961) was used as coverslip mountant. Coverslips were mounted for $24 \mathrm{~h}$ at room temperature and subjected to fluorescence microscopy.

For ethynyl uridine (EU) and 5-ethynyl-2'-deoxyuridine (EdU) incorporation

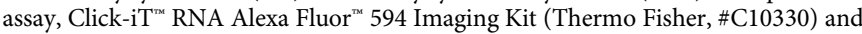
Click-iT $^{\text {ru }}$ EdU Alexa Fluor ${ }^{\text {sx }} 488$ Imaging Kit (Thermo Fisher, \#C10337) were used. Experiments were carried out following the manufacture's protocols. For EU incorporation assay, CHP-134, IMR-5, and KELLY cells were treated with CX-5461 for $24 \mathrm{~h}$. $1 \mathrm{mM}$ EU was added at $30 \mathrm{~min}$ (CHP-134, IMR-5) or $1 \mathrm{~h}$ (KELLY) before cell fixation. Nascent RNA was labeled with EU (red). Cell nuclei were stained with DAPI (blue). For EdU incorporation assay, CHP-134 and IMR-5 cells were treated with $0.2 \mu \mathrm{M}$ CX-5461 and $0.05 \mu \mathrm{M}$ CX-5461 respectively. Two hours before cell fixation, $10 \mu \mathrm{M}$ EdU was added to the medium. For EdU and $\gamma-\mathrm{H} 2 \mathrm{AX}$ colocalization, CHP-134 cells were treated with $20 \mu \mathrm{M}$ EdU and $0.2 \mu \mathrm{M}$ CX-5461 for $3 \mathrm{~h}$. IMR- 5 cells were treated with $10 \mu \mathrm{M}$ EdU and $0.1 \mu \mathrm{M}$ CX-5461 for $3 \mathrm{~h}$. Immunofluorescence was then carried out in these cells.

Briefly, cells were cultured in the medium with EU or EdU for $30 \mathrm{~min}$ to $3 \mathrm{~h}$. After fixation and permeabilization, cells were incubated for $30 \mathrm{~min}$ at room temperature in EU or EdU Click-iT ${ }^{\circledast}$ reaction cocktail and then washed in Click-iT ${ }^{\circledast}$ reaction rinse buffer (for EU) or 3\% BSA in PBS (for EdU). If needed, cells were processed for additional staining as regular immunofluorescence.

Images were taken with the Nikon C2 confocal microscope and analyzed with NIS-Elements Viewer imaging software. $\gamma$-H2AX signal were quantified and analyzed using ImageJ. The antibodies used for immunofluorescence are $\gamma-\mathrm{H} 2 \mathrm{AX}$ (Cell Signaling, \#9718; Millipore, \# 05-636; 1:500 dilution), 53BP1 (Abcam, ab36823; 1:500 dilution), RPA (Millipore, \# MABE285; 1:500 dilution), Alexa fluor 488 conjugated goat anti-mouse IgG (Thermo Fisher, \#A-11001; 1:1,000 dilution), Alexa fluor 594 conjugate anti-rabbit IgG (Cell Signaling, 8889; 1:1,000 dilution).

Comet assay. Comet assay was performed with the Comet Assay Kit (Abcam, ab238544), following the protocol from the manufacture. In brief, cells were collected and counted, then mixed with Comet Agarose at 1/10 ratio (v/v). The cell and agarose mixture was immediately transferred onto an agarose-coated glass slide. After solidified at $4{ }^{\circ} \mathrm{C}$ for $15 \mathrm{~min}$, the slide was incubated in pre-chilled Lysis Buffer for $30-60 \mathrm{~min}$ at $4{ }^{\circ} \mathrm{C}$ in the dark, then in pre-chilled Alkaline Solution for $30 \mathrm{~min}$ at $4^{\circ} \mathrm{C}$ in the dark. The slide was then subjected to electrophoresis in Alkaline Solution. After fixed in $70 \%$ ethanol, DNA was stained with Vista Green DNA Dye. Slides were viewed and imaged with Nikon C2 microscope at 10x magnification. Images were analyzed with OpenComet tool.

RADAR assay. The assay was performed as previously described ${ }^{31}$. Treated cells were lysed with $\mathrm{MB}$ buffer (6 M guanidinium isothiocyanate, $10 \mathrm{mM}$ Tris- $\mathrm{HCl}, \mathrm{pH}$ 6.5, $20 \mathrm{mM}$ EDTA, 4\% Triton X100, 1\% Sarkosyl, and 1\% dithiothreitol). Nucleic acids were precipitated by adding $100 \%$ ethanol to the cell lysate to a final concentration of $33 \%$ ( $1 / 2$ volume of the cell lysate). After $5 \mathrm{~min}$ of incubation at $-20^{\circ} \mathrm{C}$, nucleic acids were collected by centrifugation. Pellet was washed with $75 \%$ ethanol and dissolved in freshly made $8 \mathrm{mM} \mathrm{NaOH}$ solution. DNA concentration was determined by NanoDrop 1000 spectrophotometer. $1 \mu \mathrm{g}$ DNA from each sample was transferred to a nitrocellulose membrane using the Bio-Dot SF microfiltration apparatus (Bio-Rad, \#170-6542). The membrane was blocked in 5\% non-fat milk in TBS with $0.1 \%$ Tween-20, then probed with primary antibodies and horseradish-peroxidase-conjugated secondary antibodies. The signal was visualized by Western Lightning Plus-ECL (PerkinElmer). Antibodies used were listed in section 'Western blotting and band depletion assays'.

CRISPR screen. CHP-134 cells were transduced with lentiCas9-Blast lentivirus Media with $7 \mu \mathrm{g} / \mathrm{ml}$ blasticidin was added 2 days later. After 5 days of selection, a single cell was seeded to each well of a 96-well plate by flow cytometry single-cell sorting. Single clones were picked out after 3 weeks and Cas9 expression level in each clone was determined by western blotting with Cas9 antibody (Cell Signaling, \#14697). Cas9 activity was tested using the CRISPRtest ${ }^{\mathrm{Tu}}$ Functional Cas9 Activity Kit for Human Cells (Cellecta, \#CRTEST). The clone with normal viability and high Cas9 activity (> 95\%) was used for the following screening.

CHP-134 Cas9 cells were transduced at day 0 with lentiviral Brunello pooled library $^{71}$ at a low MOI to make the transduction efficiency $\sim 30 \%{ }^{72} .0 .5 \mu \mathrm{g} / \mathrm{ml}$ puromycin was used to select transductants after 2 days of transduction (day 2). Cells were subcultured on day 4 . On day 6 , cells were split into two groups, with 500 representatives for each gRNA in each group. On the next day (day 7), one group of cells were treated with $0.04 \mu \mathrm{M}$ CX-5461, DMSO was added to the other group. All cells were collected for DNA extraction after 3 days (day 10).

The Brunello sgRNA pooled plasmid library (Addgene \#73178) and lentiCas9Blast plasmid (Addgene \#52962) were expanded following manufacturer's guidelines at The Center for Advanced Genome Engineering at St. Jude Children's Research Hospital. Lentiviral particles were generated by the St. Jude Vector Development and Production Core. Genomic DNA was extracted using Nucleospin Blood XL kit (Macherey-Nagel cat\# 740950) and eluted into $1 \mathrm{ml}$ elution buffer. gDNA samples were amplified for next-generation sequencing at the St. Jude Center for Advanced Genome Engineering using primer sets and reagent recommended by the Broad Genetic Perturbation Platform (https://portals.broadinstitute.org/gpp/ public/). Samples were sequenced by the St. Jude Hartwell Center for Bioinformatics and Biotechnology and resulting sequencing data analyzed using $\mathrm{MAGeCK}^{73}$.

Animal experiments. Mice and housing conditions: Athymic nude immunodeficient mice were purchased from Charles River (strain code 553). All the mice were female and approximately 12 weeks in age. This study was carried out in strict accordance with the recommendations in the Guide to Care and Use of Laboratory Animals of the National Institute of Health. The protocol was approved by the Institutional Animal Care and Use Committee at St. Jude Children's Research Hospital. All efforts were made to minimize suffering. All mice were housed per approved IACUC protocols. Animals were housed on a 12-12 light cycle (light on 6 am off $6 \mathrm{pm}$ ) and provided food and water ad libitum.

Isolation of SJNBL046_X Cells: The tumor material was collected from a male patient diagnosed with MYCN-amplified neuroblastoma at St. Jude Children's Research Hospital, after informed consent and in agreement with local institutional ethical regulations. Tumor tissue was initially processed within $2 \mathrm{~h}$ of surgical resection into a single cell suspension by enzymatic dissociation and injected into the para-adrenal region of NSG mice using ultrasound guidance as described below. Tumor growth was monitored with ultrasound and manual palpation. After tumor development, the tumor was harvested and passaged into athymic nude mice using the same dissociation and ultrasound implantation techniques.

Isolation of SJNBL047443_X Cells: The tumor material was collected from a male patient diagnosed with ATRX-mutated neuroblastoma at St. Jude Children's Research Hospital, after informed consent and in agreement with local institutional ethical regulations. Tumor tissue was initially processed within $2 \mathrm{~h}$ of surgical resection and implanted into the flank of an NSG mouse. After tumor development, the tumor was harvested, processed into a single cell suspension by enzymatic dissociation, and passaged into athymic nude in the para-adrenal location using ultrasound guidance.

Orthotopic Ultrasound-Guided Para-adrenal Xenograft Implantation: All ultrasound procedures were performed using the VEVO 3100 high-frequency ultrasound equipped with MS-MX250 $24 \mathrm{MHz}$, MX550D $55 \mathrm{MHz}$, and MX700 $70 \mathrm{MHz}$ transducers (Fujifilm-Visualzonics, Toronto, ON, Canada). Cells were suspended in Matrigel (BD Worldwide, Cat\#354234) at a concentration of $2 \times 10^{4}$ cells per microliter and placed on ice. Anesthetized recipient athymic nude mice (isoflurane $2-3 \%$ in $100 \%$ oxygen and delivered at 2 liters $/ \mathrm{min}$ ) were placed laterally on the heated platform in right lateral recumbency. To provide a channel for delivery of the implant, a 22 gauge catheter (BD Worldwide, Cat\# 381423) was gently inserted through the skin and back muscle into the para-adrenal region, the stylet removed, and the hub clipped off. A chilled Hamilton syringe fitted with a 27 gauge needle ( 1.5 inches) and loaded with $10 \mu \mathrm{l}$ of the cell suspension was inserted using a micromanipulator through the catheter and the tip positioned between the kidney and adrenal gland via ultrasound guidance. The cell suspension was injected into the region and the needle left in place for $30 \mathrm{~s}$ to permit the Matrigel component to set before retracting the needle and catheter.

Statistical analysis: $P$-values for pairwise comparisons of all survival curves within each experiment were calculated using a log-rank test, implemented in survdiff() function in the R package "survival". Censoring plots and survival plots were created in $\mathrm{R}$ and Inkscape. 
Study 1. Neuroblastoma orthotopic xenografts were created by injecting luciferase labeled SJNBL046_X and SJNBL047443_X cells into recipient athymic nude mice using the para-adrenal injection technique previously described. Mice were screened weekly by ultrasound for tumor volume measurements. Mice were enrolled in the study when the tumor volume was the size of the adrenal gland $\left(8 \mathrm{~mm}^{3}\right)$ or above and chemotherapy was started the following Monday.

SJNBL046_X mice $(n=64)$ and SJNBL047443_X mice $(n=71)$ were randomized to nine treatment groups: liposomal irinotecan (Irin.), Irin. + CX5461, Irin. + CX-5461 + AZD6738, Irin. + CX-5461 + AZD1390, CX$5461+$ AZD1390, CX-5461 + AZD6738, and placebo. The following doses were used:

\begin{tabular}{ll}
\hline Treatment group & Dosing \\
\hline $\begin{array}{l}\text { Irin. } \\
\text { Irin. }+ \text { CX-5461 }\end{array}$ & $\begin{array}{l}5 \mathrm{mg} / \mathrm{kg} \text { once daily on days } 1 \text { and } 8 \\
\text { Irin. } 5 \mathrm{mg} / \mathrm{kg} \text { once daily on days } 1 \text { and } 8, \mathrm{CX}-54615 \mathrm{mg} / \mathrm{kg} \\
\text { once daily on days } 1-5 \text { and } 8-12 \\
\text { Irin. } 5 \mathrm{mg} / \mathrm{kg} \text { once daily on days } 1 \text { and } 8, \mathrm{CX}-54615 \mathrm{mg} / \mathrm{kg} \\
\text { once daily on days } 1-5 \text { and } 8-12, \text { AZD6738 } 50 \mathrm{mg} / \mathrm{kg} \text { once } \\
\text { Irin. }+ \text { CX- }\end{array}$ \\
$5461+$ AZD6738 on days $2,5,9,12,16$, and 19
\end{tabular}

Study 2. Neuroblastoma orthotopic xenografts were created by injecting luciferase labeled SJNBL046_X and SJNBL047443_X cells into recipient athymic nude mice using the para-adrenal injection technique previously described. Mice were screened weekly by ultrasound and tumor volume was measured. Mice were enrolled in the study when the tumor volume was the size of the adrenal gland $\left(8 \mathrm{~mm}^{3}\right)$ or above and chemotherapy was started the following Monday. Mice received 4 courses of chemotherapy and ultrasound tumor volume was monitored between courses and at the end of therapy. In addition to ultrasound, bioluminescence imaging was performed weekly. Mice were monitored daily while receiving chemotherapy and removed from the study and euthanized if found to be moribund at any time. Disease response was assigned according to tumor size. Mice that did not have a tumor at the end of the study by ultrasound and necropsy were classified as complete response. Mice that completed all four courses of therapy and had a measurable tumor less than $20 \%$ of the bodyweight were classified as a partial response. Mice with a tumor burden greater than $20 \%$ of the bodyweight at any point were removed from the study and classified as progressive disease.

SJNBL046 X mice $(n=32)$ and SJNBL047443 X mice $(n=44)$ were randomized to six treatment groups: CX-5461, liposomal irinotecan (Irin.) + temozolomide (Temo.), cyclophosphamide (Cyclo.) + topotecan (Topo.), Cyclo. + Topo. + CX-5461, Irin. + Temo. + CX-5461, and placebo. The following doses were used:

\begin{tabular}{|c|c|}
\hline Treatment group & Dosing \\
\hline $\mathrm{X}-5461$ & $5 \mathrm{mg} / \mathrm{kg}$ once daily on days $1-5$ and $8-12$ \\
\hline . & $\begin{array}{l}\text { Irin. } 5 \mathrm{mg} / \mathrm{kg} \text { once daily on days } 1 \text { and } 8 \text {, Temo. } 33 \mathrm{mg} / \mathrm{kg} \\
\text { once daily on days } 1-5\end{array}$ \\
\hline Cyclo. + Topo. & $\begin{array}{l}\text { Cyclo. } 50 \mathrm{mg} / \mathrm{kg} \text { once daily on days } 1-5 \text {, Topo. } 0.3125 \mathrm{mg} / \\
\mathrm{kg} \text { once daily on days } 1-5\end{array}$ \\
\hline $\begin{array}{l}\text { Cyclo. }+ \text { Topo. }+ \\
\text { CX-5461 }\end{array}$ & $\begin{array}{l}\text { Cyclo. } 50 \mathrm{mg} / \mathrm{kg} \text { once daily on days } 1-5 \text {, Topo. } 0.3125 \mathrm{mg} \text {, } \\
\mathrm{kg} \text { once daily on days } 1-5 \text {, CX-5461 } 5 \mathrm{mg} / \mathrm{kg} \text { once daily on } \\
\text { days } 1-5 \text { and } 8-12\end{array}$ \\
\hline $\begin{array}{l}\text { Irin. }+ \text { Temo. }+ \\
\text { CX-5461 }\end{array}$ & $\begin{array}{l}\text { Irin. } 5 \mathrm{mg} / \mathrm{kg} \text { once daily on days } 1 \text { and } 8 \text {, Temo. } 33 \mathrm{mg} / \mathrm{kg} \\
\text { once daily on days } 1-5 \text {, CX-5461 } 5 \mathrm{mg} / \mathrm{kg} \text { once daily on } \\
\text { days } 1-5 \text { and } 8-12\end{array}$ \\
\hline PLACEBO & Vehicle control (no chemotherapy) \\
\hline
\end{tabular}

Abdominal ultrasound and tumor volume acquisition: The Vevo 3100 system was used for ultrasound measurements of orthotopic neuroblastoma xenografts. Mice were anesthetized with isoflurane (2-3\% in 100\% oxygen) and positioned on the heated platform. The abdomen was coated with ultrasound gel (Aquasonics, Parker Labs, Fairfield, NI) and a MX250 or MX550 transducer used for 3D B-mode image acquisition. For volumetrics, 2D image slices were acquired in the coronal plane covering a $20-30 \mathrm{~mm}$ range with a step-size of $0.076 \mathrm{~mm}$. All postprocessing of data was performed using Vevo LAB 3.2 software. Briefly, tumor margins were manually segmented at $5-10$ slice intervals, closed at the cranial and caudal ends and reconstruction was performed to identify final volumes.
Bioluminescence imaging and quantification: Mice were anesthetized with isoflurane (2-3\% in 100\% oxygen) and bioluminescence images acquired with an IVIS Spectrum (Perkin Elmer, Waltham, MA) $5 \mathrm{~min}$ after intraperitoneal injection of Potassium D-Luciferin solution (Perkin Elmer, $3 \mathrm{mg} / \mathrm{mouse}$ ). Luminescence values for all xenografts were quantified as the average radiance (photons $/ \mathrm{s} / \mathrm{cm} 2 / \mathrm{sr}$ ) from identical regions of interest (ROI) encompassing the entire abdomen using Living Image 4.7 (Perkin Elmer).

Preclinical pharmacokinetic studies. Previously published CX-5461 dosages in mice have ranged from 12 to $50 \mathrm{mg} / \mathrm{kg}$ orally (PO) or by intraperitoneal (IP) injection, from once weekly to continuous 5-day on, 2-day off schedules $27,59,74-76$ However, the pharmacokinetic (PK) profile of CX-5461 in mice has yet to be reported in enough detail to judge the clinical relevance of such dosages and resultant plasma concentrations. To determine a PK-guided clinically relevant dosage, we measured plasma and tumor concentrations of CX-5461 in athymic nude mice at time points from $30 \mathrm{~min}$ to $24 \mathrm{~h}$ after doses of $5 \mathrm{mg} / \mathrm{kg} \mathrm{IV}$ and $25 \mathrm{mg} /$ $\mathrm{kg}$ IP (Fig. S7a-c). We then compared CX-5461 plasma area under the concentration-time curve (AUC) and average plasma concentration (Cavg) values to those reported in the available human phase I clinical trial, which established a single agent maximum tolerated dose of $170 \mathrm{mg} / \mathrm{m}^{2}$ by intravenous infusion ${ }^{15}$. We established a dosage of $5 \mathrm{mg} / \mathrm{kg}$ once daily by IP injection for 5 days, starting on day 1 , with a second 5 -day course starting on day 8 , every 21 days. Our complete PK reports for all compounds screened in this study (including CX-5461) are included as Supplementary Methods and have been made freely available on the CSTN website (https://cstn.stjude.cloud/resources and https://www.stjude.org/ content/dam/en_US/shared/www/research/cstn-appendix-standard-of-care-drugregimens.pdf).

Rescreen of GDSC cell lines with CX-5461. CX-5461 rescreening on a subset of GDSC cell lines was performed at the University of Chicago's Cellular Screening Center using the CellTiter Glo assay. We rescreened the IMR-5, CHP-134, KELLY BE(2)-M17, SK-N-SH, SK-N-FI, RS4-11, and H2452 cell lines. Cells were plated in a 96-well plate for one day then drug treated in triplicate for $72 \mathrm{~h}$ at two-fold serial dilutions at 10 concentrations from 5 to $0.000 \mu \mathrm{M}$. Luminescence was quantitated using a standard plate reader and $\mathrm{IC}_{50}$ values were estimated using a generalized logistic regression model implemented in the "drc" package in R.

High-throughput drug combination (synergy) screening. CX-5461 in combination with each of AZD6738, AZD1390, and SN-38 were dispensed into 384-well flat-bottom tissue culture-treated solid white plates (Corning, Catalog\# 3570) using a Labcyte Echo 555 acoustic Liquid Dispenser (Beckman Coulter Life Sciences, USA). Twelve-point dose-response curves were generated using 1:2 dilution intervals for each drug. The top concentrations of the drugs were derived from predetermined $\mathrm{IC}_{50}$ values and were as follows: $311.4 \mathrm{nM} \mathrm{SN}-38,1.89 \mu \mathrm{M}$ CX-5461, $19.93 \mu \mathrm{M}$ AZD6738, and 87.19 $\mu \mathrm{M}$ AZD1390. All plates contained two replicates each of the 144 pairwise combination matrices (all possible combinations of drug concentrations), the drugs alone without combination, DMSO-only (as a $0 \%$ cell death control), and the maximum concentration of each drug combined (as a 100\% cell death control). The final DMSO concentration of all wells was $0.536 \%$. Each plate was generated twice for a total of four replicates per drug combination per cell line. Into the compound-containing plates, the neuroblastoma cell lines CHP-134 (2000 cells/well), KELLY (2250 cells/well), BE(2)-M17 (1750 cells/well), and IMR-5 ( 2750 cells/well) were plated in volumes of $40 \mu \mathrm{L}$ using a Multidrop Combi reagent dispenser (ThermoFisher Scientific, USA) and settled for $20 \mathrm{~s}$ at $100 \times \mathrm{g}$ in an Eppendorf 5810 centrifuge. Plates were then incubated at $37^{\circ} \mathrm{C}$ in $5 \% \mathrm{CO}_{2}$ for $96 \mathrm{~h}$ in a Liconic STX220 incubator. Following incubation, plates were placed at room temperature for $20 \mathrm{~min}$ before viability assessment. $25 \mu \mathrm{L}$ of CellTiter-Glo (Promega Catalog\# G9241) was then added to each well to measure viability using a Multidrop Combi and the plates were incubated for an additional $25 \mathrm{~min}$ at room temperature. Luminescence was then detected with an EnVision 2102 Multilabel Plate Reader (PerkinElmer Life Sciences, USA).

Drug synergy analysis. Raw luminescent data were imported into the R statistical environment version 4.0.2 (www.r-project.org). Background-subtracted values in raw luminescent units (RLU) were assigned to the appropriate drugs and concentrations, and each replicate was separated. All replicates were normalized to the mean of their respective inter-plate controls (DMSO for $0 \%$ cell death, and the maximum concentration of each drug combined for $100 \%$ cell death). Normalized drug-only data were fit with log-logistic regression to produce dose-response curves using the packages $\mathrm{drc}^{77}$ and tidydrc (https://github.com/angelovangel/ tidydrc). $\mathrm{EC}_{50}$ values for each drug's curve were extrapolated and plotted. Matrices of the percent cell death values were constructed using means of normalized data from each of the four replicates per group as input. From these normalized values, synergy scores were calculated for all tested concentration combinations. Delta scores derived from the Zero Interaction Potency (ZIP) model $^{49}$ were used to score synergy across all samples using the SynergyFinder package ${ }^{78}$. The most significant synergy (or antagonism) scores of drug pairs were directly compared to their corresponding single agents by referencing original normalized data of individual replicates and plotting mean values of percent cell death. From the resulting 
synergy matrices, both the highest- and lowest-scoring concentration pairs were extracted to represent the most significant synergy and antagonism, respectively. These scores were used to reference the expected percent cell death of a given combination (based on assumed non-interaction) to test whether the differences were statistically significant. $P$-values were calculated using a one sample t-test.

TOP2A and TOP2B activity assays. Decatenation assay: $1 \mathrm{U}$ of human topoisomerase II was incubated with $200 \mathrm{ng} \mathrm{kDNA}$ in a $30 \mu \mathrm{l}$ reaction at $37 \mathrm{C}$ for $30 \mathrm{~min}$ under the following conditions: $50 \mathrm{mM}$ Tris $\mathrm{HCl}(\mathrm{pH} 7.5), 125 \mathrm{mM} \mathrm{NaCl}, 10 \mathrm{mM}$ $\mathrm{MgCl} 2,5 \mathrm{mM}$ DTT, $0.5 \mathrm{mM}$ EDTA, $0.1 \mathrm{mg} / \mathrm{ml}$ bovine serum albumin (BSA) and $1 \mathrm{mM}$ ATP. Each reaction was stopped by the addition of $30 \mu \mathrm{l}$ chloroform/isoamyl alcohol (24:1) and $30 \mu \mathrm{l}$ Stop Dye before being loaded on a 1.0\% TAE gel run at $90 \mathrm{~V}$ for $90 \mathrm{~min}$. Bands were visualized by ethidium bromide staining for $15 \mathrm{~min}$ and destaining for $10 \mathrm{~min}$. Gels were scanned using documentation equipment (GeneGenius, Syngene, Cambridge, UK) and decatenation levels were calculated from band data obtained using ImageJ. A second individual also quantitated these images independently using different gel scanning software (GeneTools, Syngene, Cambridge, UK) and obtained similar results. The activity of the enzymes was determined before the testing of the compound. The amount of topoisomerase II required for optimal decatenation was determined by titration. The controls and compounds were tested over a range of dilutions from 1.734 to $400 \mu \mathrm{M}$ and added to the reaction before the addition of the enzyme. Final DMSO concentration in the assays was $10 \%(\mathrm{v} / \mathrm{v})$. All experiments were performed five times and run on $1 \%$ agarose gels. Dose-response curves were estimated using a generalized logistic regression model fit to the data using the R package "drc", from which $\mathrm{IC}_{25} \mathrm{~s}$ and $95 \%$ confidence intervals were also calculated. Note that $\mathrm{IC}_{25} \mathrm{~s}$ were calculated because a measurable $\mathrm{IC}_{50}$ was not achieved within the active drug concentration range for CX-5461 in the TOP2A assays; to fit the dose-response curves properly it was also necessary to filter those data, removing points beyond the activity range of CX-5461.

Relaxation Assays: $1 \mathrm{U}$ of human topo II was incubated with $500 \mathrm{ng}$ supercoiled pBR322 in a $30 \mu \mathrm{l}$ reaction at $37 \mathrm{C}$ for $30 \mathrm{~min}$ under the following conditions: $50 \mathrm{mM}$ Tris $\mathrm{HCl}$ (pH 7.5), $125 \mathrm{mM} \mathrm{NaCl}, 10 \mathrm{mM} \mathrm{MgCl} 2,5 \mathrm{mM}$ DTT, $0.5 \mathrm{mM}$ EDTA, $0.1 \mathrm{mg} / \mathrm{ml}$ bovine serum albumin (BSA) and $1 \mathrm{mM}$ ATP. Each reaction was stopped by the addition of $30 \mu \mathrm{l}$ chloroform/iso-amyl alcohol (24:1) and $30 \mu \mathrm{l}$ Stop Dye before being loaded on a $1.0 \%$ TAE gel run at $90 \mathrm{~V}$ for $90 \mathrm{~min}$. Bands were visualized by ethidium bromide staining for $15 \mathrm{~min}$ and destaining for $10 \mathrm{~min}$. Gels were scanned using documentation equipment (GeneGenius, Syngene, Cambridge, UK) and relaxation levels were calculated from band data obtained with gel scanning software (GeneTools, Syngene, Cambridge, UK). The data for all topoisomerase activity assays were generated by Inspiralis (Innovation Center, Norwich Research Park, Colney Lane, Norwich, UK) and the experimental details above were provided by Inspiralis. The data were analyzed by Inspiralis and reanalyzed by M.P and P.G, achieving consistent results.

CX-5461-Diazirine crosslinking and affinity purification. CHP-134 cells were grown in four $15 \mathrm{~cm}$ dishes and treated with $1 \mu \mathrm{M} \mathrm{CX}-5461$-Diazirine for $24 \mathrm{~h}$. Following CX-5461-Diazirine treatment, growth media was replaced, and cells were photo-crosslinked as previously described (Erwin et al. JOVE 2016) for $20 \mathrm{~min}$. Cells were rapidly scraped in $37^{\circ} \mathrm{C}$ PBS and pooled. Cell lysis was performed in $200 \mu \mathrm{L}$ RIPA buffer using Tris buffer $\mathrm{pH} 8$ and supplemented with $4 \mathrm{U}$ Turbo DNAse (Invitrogen, \#AM2238). After resuspending cells on ice, lysate was immediately sonicated at room temperature for $20 \mathrm{~min}$ and then precleared for $10 \mathrm{~min}$ at $4^{\circ} \mathrm{C}, 18,400 \times g$. Lysate was split into 2 click chemistry reactions using the Click-\&-Go Click Chemistry Reaction Kit (Click Chemistry Tools, \#1001) in the presence or absence of N-1-(4,4-dimethyl-2,6-dioxocyclohexylidene) ethyl (Dde) Biotin Azide (Click Chemistry Tools, \#1136) and incubated for $90 \mathrm{~min}$. To remove free biotin, click chemistry reactions were run through a $7 \mathrm{kDa}$ Zeba Spin Desalting Column (ThermoFisher, \#89892), followed by three centrifugations through an Amicon Ultra Centrifugal $3 \mathrm{~K}$ Filter spin concentrator (Millipore, \# UFC500324), both pre-equilibrated with lysis buffer.

For affinity purification of biotinylated CX-5461-Diazirine, $600 \mu \mathrm{L}(300 \mu \mathrm{L}$ per pulldown) of Dynabeads M-280 Streptavidin (Invitrogen, \# 11206D) were washed two times in lysis buffer and then resuspended in the spin-concentrated click reactions, followed by rotation at room temperature for $2 \mathrm{~h}$. Beads were then washed five times with $25 \mathrm{mM}$ Tris buffer $\mathrm{pH} 8,500 \mathrm{mM} \mathrm{NaCl}, 4 \mathrm{M}$ Urea, $1 \% \mathrm{w} / \mathrm{v}$ sodium dodecyl sulfate (SDS) followed by 1 wash in lysis buffer. To elute crosslinked material, beads were then resuspended in $20 \mu \mathrm{L} 2 \% \mathrm{v} / \mathrm{v}$ hydrazine (Sigma-Aldrich, \#215155) in $\mathrm{H}_{2} \mathrm{O}$ and rotated for $1 \mathrm{~h}$ at room temperature. The hydrazide-liberated material was then added to $20 \mu \mathrm{L} 2 \mathrm{X}$ Laemli Buffer (Bio Rad, \#1610737). For western blot, samples were separated by $4-20 \%$ SDSpolyacrylamide gel electrophoresis, electrophoretically transferred to nitrocellulose, and probed for TOP2B using a 1:500 dilution of mouse anti-TOP2B antibody solution in 5\% bovine serum albumin in Tris Buffered Saline (TBS) overnight at $4{ }^{\circ} \mathrm{C}$. The membrane was then washed with TBS $+0.1 \% \mathrm{v} / \mathrm{v}$ Tween- 20 four times for 5 min each and then incubated with anti-mouse secondary antibody

(Invitrogen, \#31430) at a 1:2500 dilution in 5\% milk + TBS $+0.1 \% \mathrm{v} / \mathrm{v}$ Tween-20 for $2 \mathrm{~h}$ at room temperature. The membrane was then washed with TBS $+0.1 \% \mathrm{v} / \mathrm{v}$ Tween-20 four times for $5 \mathrm{~min}$ each. Chemiluminescent signal was detected using Amersham ECL Select (GE Healthcare, \# RPN2235).
Ligand docking and molecular dynamics simulations. Published crystal structures of etoposide-bound TOP2A (PDB: 5GWK) or TOP2B (PDB: 3QX3) were loaded into Maestro software (Schrödinger Release 2020-3). To prepare the protein for docking and simulations, the protein preparation wizard was used to assign bond orders, add hydrogens, create zero-order bonds to metals, create disulfide bonds, and fill in missing side chains and loops. Default parameters were used for the optimization of hydrogen-bond assignment (sampling of water orientations and use of $\mathrm{pH}$ 7.0). Waters beyond $5 \AA$ of het groups or with fewer than three hydrogen bonds to non-waters were removed. Restrained energy minimization was applied using the OPLS3e forcefield. Prepared protein systems were further checked by Ramachandran plots, ensuring there were no steric clashes.

For docking CX-5461 into TOP2A and TOP2B, the 3D structure of CX-5461 was first obtained from the PubChem database (www.pubchem.ncbi.nlm.nih.gov) The flexible ligand alignment tool in Maestro was used to align the core scaffold of CX-5461 to etoposide based on common scaffolds defined by the Bermis-Murcko method. The aligned molecule was then translated into the etoposide bound site based on these aligned scaffolds. The ligand position was adjusted in the binding site so that CX-5461 would occupy the optimal volume of the site through rigid body minimization of CX-5461 using Prime. The Prime tool was further used to adjust protein sidechain rotamers of TOP2A and TOP2B that are within $5 \AA$ of CX-5461. The binding site was further optimized by administering a conjugategradient minimization algorithm for all residues within $5 \AA$ of CX-5461, with a $200 \mathrm{kcal} / \mathrm{mol} / \AA^{2}$ restraint placed on a shell of residues greater than $5 \AA$ but less than $7 \AA$ from CX-5461. All atoms greater than $7 \AA$ from CX-5461 were ignored by the algorithm.

For molecular dynamics simulations, systems were built for CX-5461-bound TOP2A and TOP2B by using the system builder panel of Desmond (Schrödinger Release 2020-3). The SPC solvent model was used, and the forcefield was set to OPLS3e. Solvated systems were loaded into the workspace by using the molecular dynamics panel. The total simulation time for each system was set to 10 nanoseconds, with 100-picosecond trajectory recording intervals. The system energy was set to 1.2 , and the ensemble class used was NPT. Simulations were set to run at $300.0 \mathrm{~K}$ and 1.01325 bar. The option to relax model systems before simulations were selected.

Simulations were clustered based on RMSD using default parameters in the tri_cluster.py command line script available in Schrodinger utilities. This resulted in 12 clusters for TOP2A and 16 clusters for TOP2B. For each cluster, the representative structure file was used to calculate the relative binding affinity of TOP2A and TOP2B to the CX-5461 ligand. The binding energy was calculated using the Prime MM-GBSA tool from the Maestro GUI. The population of each cluster was used to determine weights for calculating a weighted average binding energy for TOP2A and TOP2B with CX-5461 from each of the clusters of the trajectory.

Cell line drug screening analysis. Preprocessed GDSC data (drug sensitivity, gene expression, and mutation calls) were obtained from the GDSC website (www.cancerrxgene.org). All analyses of these data were performed using R and figures were created using the base graphics functions and the ggplot2 package. Basic statistical tests such as t-tests, linear regression, Wilcoxon-rank sum tests, and correlation tests were performed using the base statistical functions in $\mathrm{R}$, and where appropriate, $P$-values were corrected for multiple testing by estimating falsediscovery rates using the Benjamini and Hochberg method. The PRISM drug screening data were obtained from www.depmap.org.

Patient -omics data analysis. Neuroblastoma patient tumor data was obtained from Brady et al. ${ }^{39}$ who aggregated data from multiple previously published neuroblastoma studies including RNA-seq, clinical annotations, and genomics sequencing data. The associations between TOP2B expression and individual genomic and clinical features were estimated using linear regression. Sparse regression models, aimed at identifying independent predictors of TOP $2 B$ expression were fit using ElasticNet regression, implemented in the $\mathrm{R}$ package glmnet, where the alpha parameter was fixed to 0.5 and the lambda parameter was deduced by 10 -fold cross-validation. GTEx data were obtained from the GTEx data portal (www.gtexportal.org), TCGA data from the Genomics Data Commons, paired relapse data was obtained from TARGET ${ }^{79}$, and data from the paired primary tumor and metastatic sites was obtained from Rifatbegovic et al. ${ }^{40}$. Paired patient tumor-PDX gene expression data for our 2 xenografts was obtained from the St. Jude Cloud ${ }^{80}$. When comparing the expression of TOP $2 B$, we also normalized these gene expression data by calculating their $\log _{2}$ fold-change relative to housekeeping genes ACTIN and GAPDH, which are treated as negative controls whose expression is not expected to vary, thus mitigating the effect of gene expression differences that would be expected to arise due to dataset-specific effects.

Computational alignment of cell lines and tumor samples. To align cell lines to their corresponding lineages of patient tumor samples, the Celligner tool ${ }^{37}$ was used. Clinical expression data were obtained from the UCSC Xena data hub (www.xena. ucsc.edu), which included processed data for tumor samples originating from The Cancer Genome Atlas (TCGA, www.cancer.gov) and TARGET (www.ocg.cancer.gov) 
datasets. Cell line expression data were obtained from Expression Atlas (www.ebi.ac.uk), which included RNA-sequencing of cell lines used in the Sanger Genomics of Drug Sensitivity in Cancer (GDSC, www.cancerrxgene.org) project. All expression data were in units of $\log _{2}(\mathrm{TPM}+1)$ and datasets were filtered such that only common lineages between tumors and cell lines were kept and used as input. After completion of the Celligner tool and the resulting UMAP plot was generated, alignments of cell lines to tumors were quantified by their respective lineages. Briefly, Celligner-generated tumor clusters were isolated and kept if they represented $\geq 80 \%$ of a given lineage. Proportions of cell lines that correctly aligned to their tumor counterpart clusters (by lineage) were then calculated. Fisher exact tests were performed to assess statistical significance for all alignments, and the resulting $P$-values and odds ratios were generated for each lineage. Step-by-step details of the Celligner tool, post-alignment analysis, and all input data are available in the Open Science Framework (www.osf.io/pzke4).

Reporting summary. Further information on research design is available in the Nature Research Reporting Summary linked to this article.

\section{Materials availability}

PDX models that were used in this study are freely available upon request from the Childhood Solid Tumor Network (CSTN), which is hosted at St. Jude (https:// www.stjude.org/research/resources-data/childhood-solid-tumor-network/availableresources.html). We have also made the full detailed pharmacokinetic reports for the compounds used in our PDX studies (including CX-5461) available on the CSTN website (https://cstn.stjude.cloud/resources and https://www.stjude.org/content/dam/en_US/ shared/www/research/cstn-appendix-standard-of-care-drug-regimens.pdf). Source data are provided with this paper.

\section{Data availability}

The main data generated in this study are included in the article and its Supplementary files. The source data for Figs. 1-4, 7 and Supplementary Figs. 1-4, 6, 7 are provided in the Source Data file. Drug screening and gene expression data for Figs. 1a-c, f, l, m, 4c, d and Supplementary Fig. 1a, e, f are from GDSC [https://www.cancerrxgene.org/ bulk_download]. Drug screening and gene expression data for Figs. 1d, e, 4a, b, 5c and Supplementary Figs. 1d, 4a, b, 5g are from DepMap [https://depmap.org/portal/ download/all/]. Gene expression data for Fig. 5a, b and Supplementary Fig. 5a are from UCSC Xena repository [xenabrowser.net]. Gene expression data for Fig. 5d, g, h and Supplementary Fig. 5b, c are from TCGA [https://firebrowse.org]. Gene expression data for Fig. $5 \mathrm{f}$ are from TARGET [https://target-data.nci.nih.gov/Public/NBL/mRNA-seq/L3/ expression/NCI-Khan/]. Gene expression data for Fig. $5 \mathrm{i}$ and Supplementary Fig. 5e, fare from GTEx [https://gtexportal.org/home/datasets]. Further information of the data sources is stated in figure legends and Methods. Details of additional data that support the findings of this study will be available upon reasonable request. Source data are provided with this paper.

\section{Code availability}

The code to reproduce computational analyses in this study, including the supporting data, have been deposited on Open Science Framework (osf.io/pzke4/).

Received: 15 March 2021; Accepted: 13 October 2021; Published online: 09 November 2021

\section{References}

1. Louis, C. U. \& Shohet, J. M. Neuroblastoma: molecular pathogenesis and therapy. Annu. Rev. Med. 66, 49-63 (2015).

2. Ma, X. et al. Pan-cancer genome and transcriptome analyses of 1,699 paediatric leukaemias and solid tumours. Nature 555, 371-376 (2018).

3. Wedekind, M. F., Denton, N. L., Chen, C.-Y. \& Cripe, T. P. Pediatric cancer immunotherapy: opportunities and challenges. Pediatr. Drugs 20, 395-408 (2018).

4. Moreno, L. et al. Accelerating drug development for neuroblastoma: summary of the second neuroblastoma drug development Strategy forum from innovative therapies for children with cancer and international society of paediatric oncology Europe neuroblastoma. Eur. J. Cancer 136, 52-68 (2020).

5. Barretina, J. et al. The Cancer Cell Line Encyclopedia enables predictive modelling of anticancer drug sensitivity. Nature 483, 603-607 (2012).

6. Garnett, M. J. et al. Systematic identification of genomic markers of drug sensitivity in cancer cells. Nature 483, 570-575 (2012).

7. Federico, S. M. et al. A phase I trial of talazoparib and irinotecan with and without temozolomide in children and young adults with recurrent or refractory solid malignancies. Eur. J. Cancer 137, 204-213 (2020).

8. Stewart, E. et al. Targeting the DNA repair pathway in Ewing sarcoma. Cell Rep. 9, 829-840 (2014).
9. Kim, K. H. \& Roberts, C. W. Targeting EZH2 in cancer. Nat. Med. 22, 128-134 (2016).

10. Durbin, A. D. et al. Selective gene dependencies in MYCN-amplified neuroblastoma include the core transcriptional regulatory circuitry. Nat. Genet. 50, 1240-1246 (2018).

11. Puissant, A. et al. Targeting MYCN in neuroblastoma by BET bromodomain inhibition. Cancer Discov. 3, 308-323 (2013).

12. Behan, F. M. et al. Prioritization of cancer therapeutic targets using CRISPR-Cas9 screens. Nature 568, 511-516 (2019).

13. Corsello, S. M. et al. Discovering the anticancer potential of non-oncology drugs by systematic viability profiling. Nat. Cancer 1, 235-248 (2020).

14. Francies, H. E., McDermott, U. \& Garnett, M. J. Genomics-guided pre-clinical development of cancer therapies. Nat. Cancer 1, 482-492 (2020).

15. Khot, A. et al. First-in-human RNA polymerase I transcription inhibitor CX5461 in patients with advanced hematologic cancers: results of a phase I doseescalation study. Cancer Discov. 9, 1036-1049 (2019).

16. Austin, C. A. et al. TOP2B: the first thirty years. Int. J. Mol. Sci. 19, 2765 (2018).

17. Cowell, I. G. et al. Model for MLL translocations in therapy-related leukemia involving topoisomerase II $\beta$-mediated DNA strand breaks and gene proximity. Proc. Natl Acad. Sci. USA 109, 8989-8994 (2012).

18. Zhang, S. et al. Identification of the molecular basis of doxorubicin-induced cardiotoxicity. Nat. Med. 18, 1639-1642 (2012).

19. Iorio, F. et al. A landscape of pharmacogenomic interactions in cancer. Cell 166, 740-754 (2016).

20. Liu, X., Turbyville, T., Fritz, A. \& Whitesell, L. Inhibition of insulin-like growth factor I receptor expression in neuroblastoma cells induces the regression of established tumors in mice. Cancer Res. 58, 5432-5438 (1998).

21. Bywater, M. J. et al. Inhibition of RNA polymerase I as a therapeutic strategy to promote cancer-specific activation of p53. Cancer Cell 22, 51-65 (2012).

22. Hald, $\varnothing$. H. et al. Inhibitors of ribosome biogenesis repress the growth of MYCN-amplified neuroblastoma. Oncogene 38, 2800-2813 (2019).

23. Olivieri, M. et al. A genetic map of the response to DNA damage in human cells. Cell 182, 481-496. e421 (2020).

24. Ledesma, F. C., El Khamisy, S. F., Zuma, M. C., Osborn, K. \& Caldecott, K. W. A human 5'-tyrosyl DNA phosphodiesterase that repairs topoisomerasemediated DNA damage. Nature 461, 674-678 (2009).

25. Morimoto, S. et al. Type II DNA topoisomerases cause spontaneous doublestrand breaks in genomic DNA. Genes 10, 868 (2019).

26. Crawford, L. J., Anderson, G., Johnston, C. K. \& Irvine, A. E. Identification of the APC/C co-factor FZR1 as a novel therapeutic target for multiple myeloma. Oncotarget 7, 70481 (2016).

27. Haddach, M. et al. Discovery of CX-5461, the first direct and selective inhibitor of RNA polymerase I, for cancer therapeutics. ACS Med. Chem. Lett. 3, 602-606 (2012).

28. Levine, C., Hiasa, H. \& Marians, K. J. DNA gyrase and topoisomerase IV: biochemical activities, physiological roles during chromosome replication, and drug sensitivities. Biochim. Biophys. Acta (BBA)-Gene Struct. Expr. 1400, 29-43 (1998).

29. Liang, X. et al. A comprehensive review of topoisomerase inhibitors as anticancer agents in the past decade. Eur. J. Med. Chem. 171, 129-168 (2019).

30. Kaufmann, S. H. \& Svingen, P. A. in DNA Topoisomerase Protocols, 253-268 (Springer, 1999)

31. Kiianitsa, K. \& Maizels, N. A rapid and sensitive assay for DNA-protein covalent complexes in living cells. Nucleic Acids Res. 41, e104-e104 (2013).

32. Tammaro, M., Barr, P., Ricci, B. \& Yan, H. Replication-dependent and transcription-dependent mechanisms of DNA double-strand break induction by the topoisomerase 2-targeting drug etoposide. PLoS One 8, e79202 (2013).

33. Bruno, P. M. et al. The primary mechanism of cytotoxicity of the chemotherapeutic agent CX-5461 is topoisomerase II poisoning. Proc. Natl Acad. Sci. USA 117, 4053-4060 (2020).

34. Nitiss, J. L. Targeting DNA topoisomerase II in cancer chemotherapy. Nat. Rev. Cancer 9, 338-350 (2009).

35. Prakash, V. et al. Ribosome biogenesis during cell cycle arrest fuels EMT in development and disease. Nat. Commun. 10, 1-16 (2019).

36. Hsiao, C. J. et al. Characterizing and inferring quantitative cell cycle phase in single-cell RNA-seq data analysis. Genome Res. 30, 611-621 (2020).

37. Warren, A. et al. Global computational alignment of tumor and cell line transcriptional profiles. Nat. Commun. 12, 1-12 (2021).

38. Tsherniak, A. et al. Defining a cancer dependency map. Cell 170, 564-576. e516 (2017)

39. Brady, S. W. et al. Pan-neuroblastoma analysis reveals age-and signatureassociated driver alterations. Nat. Commun. 11, 1-13 (2020).

40. Rifatbegovic, F. et al. Neuroblastoma cells undergo transcriptomic alterations upon dissemination into the bone marrow and subsequent tumor progression. Int. J. Cancer 142, 297-307 (2018).

41. Nusinow, D. P. et al. Quantitative proteomics of the cancer cell line encyclopedia. Cell 180, 387-402.e316 (2020). 
42. Negi, S. S. \& Brown, P. Transient rRNA synthesis inhibition with CX-5461 is sufficient to elicit growth arrest and cell death in acute lymphoblastic leukemia cells. Oncotarget 6, 34846 (2015).

43. Yan, S. et al. Targeting RNA Polymerase I transcription synergises with TOP1 inhibition in potentiating the DNA damage response in high-grade serous ovarian cancer. Br. J. Cancer 124, 616-627 (2021).

44. Durant, S. T. et al. The brain-penetrant clinical ATM inhibitor AZD1390 radiosensitizes and improves survival of preclinical brain tumor models. Sci. Adv. 4, eaat1719 (2018).

45. Foote, K. M. et al. Discovery and Characterization of AZD6738, a Potent Inhibitor of Ataxia Telangiectasia Mutated and Rad3 Related (ATR) Kinase with Application as an Anticancer Agent. J. Med. Chem. 22, 9889-9907 (2018).

46. Liu, L. F. et al. Mechanism of action of camptothecin. Ann. N. Y. Acad. Sci. 922, 1-10 (2000).

47. Vanhoefer, U. et al. Irinotecan in the treatment of colorectal cancer: clinical overview. J. Clin. Oncol. 19, 1501-1518 (2001).

48. Vendetti, F. P. et al. The orally active and bioavailable ATR kinase inhibitor AZD6738 potentiates the anti-tumor effects of cisplatin to resolve ATMdeficient non-small cell lung cancer in vivo. Oncotarget 6, 44289 (2015).

49. Yadav, B., Wennerberg, K., Aittokallio, T. \& Tang, J. Searching for drug synergy in complex dose-response landscapes using an interaction potency model. Comput. Struct. Biotechnol. J. 13, 504-513 (2015).

50. DuBois, S. G. et al. Phase II trial of alisertib in combination with irinotecan and temozolomide for patients with relapsed or refractory neuroblastoma. Clin. Cancer Res. 24, 6142-6149 (2018).

51. Federico, S. M. et al. A pilot trial of humanized anti-GD2 monoclonal antibody (hu14. 18K322A) with chemotherapy and natural killer cells in children with recurrent/refractory neuroblastoma. Clin. Cancer Res. 23, 6441-6449 (2017).

52. Stokes, J. M. \& Brown, E. D. Chemical modulators of ribosome biogenesis as biological probes. Nat. Chem. Biol. 11, 924-932 (2015).

53. Hein, N., Hannan, K. M., D'Rozario, J. \& Hannan, R. Inhibition of Pol I Transcription a New Chance in the Fight Against Cancer. Technol. Cancer Res. Treat. 16, 736-739 (2017).

54. Raje, S. \& Barthwal, R. Molecular recognition of $3+1$ hybrid human telomeric G-quadruplex DNA d-[AGGG (TTAGGG) 3] by anticancer drugs epirubicin and adriamycin leads to thermal stabilization. Int. J. Biol. Macromol. 139, 1272-1287 (2019).

55. Raje, S., Pandav, K. \& Barthwal, R. Dual mode of binding of anti cancer drug epirubicin to G-quadruplex [d-(TTAGGGT)] 4 containing human telomeric DNA sequence induces thermal stabilization. Bioorg. Med. Chem. 27, 115131 (2019).

56. Raje, S., Pandav, K. \& Barthwal, R. Binding of anticancer drug adriamycin to parallel G-quadruplex DNA [d-(TTAGGGT)] 4 comprising human telomeric DNA leads to thermal stabilization: a multiple spectroscopy study. J. Mol. Recogn. 33, e2815 (2020).

57. Tripathi, S. \& Barthwal, R. NMR based structure reveals groove binding of mitoxantrone to two sites of [d-(TTAGGGT)] 4 having human telomeric DNA sequence leading to thermal stabilization of G-quadruplex.Int. J. Biol. Macromol. 111, 326-341 (2018).

58. Wang, Y. et al. G-quadruplex DNA drives genomic instability and represents a targetable molecular abnormality in ATRX-deficient malignant glioma. Nat. Commun. 10, 1-14 (2019)

59. $\mathrm{Xu}, \mathrm{H}$. et al. CX-5461 is a DNA G-quadruplex stabilizer with selective lethality in BRCA1/2 deficient tumours. Nat. Commun. 8, 1-18 (2017).

60. Bossaert, M. et al. Transcription-associated topoisomerase $2 \alpha$ (TOP2A) activity is a major effector of cytotoxicity induced by G-quadruplex ligands. eLife 10, e65184 (2021)

61. Ide, S., Imai, R., Ochi, H. \& Maeshima, K. Transcriptional suppression of ribosomal DNA with phase separation. Sci. Adv. 6, eabb5953 (2020).

62. Mars, J.-C. et al. The chemotherapeutic agent CX-5461 irreversibly blocks RNA polymerase I initiation and promoter release to cause nucleolar disruption, DNA damage, and cell inviability. NAR Cancer 2, zcaa032 (2020).

63. Pommier, Y., Sun, Y., Shar-yin, N. H. \& Nitiss, J. L. Roles of eukaryotic topoisomerases in transcription, replication and genomic stability. Nat. Rev. Mol. Cell Biol. 17, 703 (2016).

64. Zhang, H., Wang, J. C. \& LIu, L. F. Involvement of DNA topoisomerase I in transcription of human ribosomal RNA genes. Proc. Natl Acad. Sci. USA 85, 1060-1064 (1988).

65. Burger, K. et al. Chemotherapeutic drugs inhibit ribosome biogenesis at various levels. J. Biol. Chem. 285, 12416-12425 (2010).

66. Leach, D. DNA Topology and Its Biological Effects, Monograph 20. Edited by Nicholas R. Cozzarelli and James C. Wang. Cold Spring Harbor Laboratory1990. 480 pages. Price \$97. ISBN 087969348 7. Genet. Res. 57, 284 (1991).

67. Uusküla-Reimand, L. et al. Topoisomerase II beta interacts with cohesin and CTCF at topological domain borders. Genome Biol. 17, 1-22 (2016).
68. Sanij, E. et al. CX-5461 activates the DNA damage response and demonstrates therapeutic efficacy in high-grade serous ovarian cancer. Nat. Commun. 11, 1-18 (2020).

69. Yang, X., Li, W., Prescott, E. D., Burden, S. J. \& Wang, J. C. DNA topoisomerase II $\beta$ and neural development. Science 287, 131-134 (2000).

70. Frank, B. Y. et al. Characterization of the phototoxicity, chemigenetic profile, and mutational signatures of the chemotherapeutic CX-5461 in Caenorhabditis elegans. Genetics 215, 609-621 (2020).

71. Doench, J. G. et al. Optimized sgRNA design to maximize activity and minimize off-target effects of CRISPR-Cas9. Nat. Biotechnol. 34, 184-191 (2016).

72. Doench, J. G. Am I ready for CRISPR? A user's guide to genetic screens. Nat. Rev. Genet. 19, 67 (2018)

73. $\mathrm{Li}, \mathrm{W}$. et al. MAGeCK enables robust identification of essential genes from genome-scale CRISPR/Cas9 knockout screens. Genome Biol. 15, 1-12 (2014).

74. Drygin, D. et al. Targeting RNA polymerase I with an oral small molecule CX5461 inhibits ribosomal RNA synthesis and solid tumor growth. Cancer Res. 71, 1418-1430 (2011).

75. Li, L. et al. CX-5461 induces autophagy and inhibits tumor growth via mammalian target of rapamycin-related signaling pathways in osteosarcoma. OncoTargets Ther. 9, 5985 (2016)

76. Maclachlan, K. H. et al. Novel combination therapies with the RNA polymerase I inhibitor CX-5461 significantly improve efficacy in multiple myeloma. Blood 130, 1805-1805 (2017).

77. Ritz, C., Baty, F., Streibig, J. C. \& Gerhard, D. Dose-response analysis using R. PLoS One 10, e0146021 (2015).

78. Ianevski, A., He, L., Aittokallio, T. \& Tang, J. SynergyFinder: a web application for analyzing drug combination dose-response matrix data. Bioinformatics $\mathbf{3 3}$, 2413-2415 (2017)

79. Pugh, T. J. et al. The genetic landscape of high-risk neuroblastoma. Nat. Genet. 45, 279-284 (2013)

80. McLeod, C. et al. St. Jude Cloud: a pediatric cancer genomic data-sharing ecosystem. Cancer Discov. 11, 1082-1099 (2021).

81. Behan, F. M. et al. Prioritization of cancer therapeutic targets using CRISPRCas9 screens. Nature 568, 511-516 (2019)

\section{Acknowledgements}

P.G. is supported by the NIH, including an R00 award from NHGRI (5R00HG009679 03), an R35 award from NIGMS (1R35GM138293-01), and an R01 award from the NCI (1R01CA260060-01). S.C.B. is partially supported by NIH grants 2R01GM079238 and RM1HG011563. The content is solely the responsibility of the authors and does not necessarily represent the official views of the National Institutes of Health. P.G. also receives funding from ALSAC. The authors acknowledge Dr. Jinghui Zhang and Dr. Alex Gout for critical review of the manuscript, and Melissa D. Johnson, Thomas Confer, and Walter J. Akers for performing the in vivo imaging work.

\section{Author contributions}

P.G. conceived and directed the study. M.P. performed the experimental work. P.G. and M.P. wrote the manuscript. P.G. and J.E. supervised the experimental work. C.W., R.C., M.S., A.Z., S.B., D.M., M.P., and P.G. performed computational work. J.B. performed the photo-crosslinking experiments and assisted with topoisomerase activity assays, supervised by S.C.B. B.C.H made the diazirine-CX5461 derivative. J.L. and S.C. performed drug screening, with C.W. analyzing the data. Y.W. performed pharmacokinetic and bioanalytical assays, supervised by B.B.F. K.B.B., B.G., P.A., and E.S. performed the PDX experiments, directed by E.S., P.C., and S.M. P.M assisted with CRISPR screening. J.B., S.N., and M.J.P. performed additional supportive experimental work. R.K., M.M.B., M.A.D., J.S., T.C., L.A.G., and S.B provided resources, insights and supervised additional parts of the study. All authors edited the final manuscript.

\section{Competing interests}

The authors declare no competing interests.

\section{Additional information}

Supplementary information The online version contains supplementary material available at https://doi.org/10.1038/s41467-021-26640-x.

Correspondence and requests for materials should be addressed to John Easton or Paul Geeleher.

Peer review information Nature Communications thanks Keefe Chan, William Weiss, and the other anonymous reviewer(s) for their contribution to the peer review this work.

Reprints and permission information is available at http://www.nature.com/reprints

Publisher's note Springer Nature remains neutral with regard to jurisdictional claims in published maps and institutional affiliations. 
(c) (i) Open Access This article is licensed under a Creative Commons Attribution 4.0 International License, which permits use, sharing, adaptation, distribution and reproduction in any medium or format, as long as you give appropriate credit to the original author(s) and the source, provide a link to the Creative Commons license, and indicate if changes were made. The images or other third party material in this article are included in the article's Creative Commons license, unless indicated otherwise in a credit line to the material. If material is not included in the article's Creative Commons license and your intended use is not permitted by statutory regulation or exceeds the permitted use, you will need to obtain permission directly from the copyright holder. To view a copy of this license, visit http://creativecommons.org/ licenses/by/4.0/.

(C) The Author(s) 2021 